Jean-Marc Bardet* and Béchir Dola

\title{
Semiparametric Stationarity and Fractional Unit Roots Tests Based on Data-Driven Multidimensional Increment Ratio Statistics
}

DOI 10.1515/jtse-2014-0031

Published online April 12, 2016

Abstract: In this paper, we show that the central limit theorem (CLT) satisfied by the data-driven Multidimensional Increment Ratio (MIR) estimator of the memory parameter $d$ established in Bardet and Dola (2012. Adaptive Estimator of the Memory Parameter and Goodness-of-Fit Test Using a Multidimensional Increment Ratio Statistic." Journal of Multivariate Analysis 105:222-40) for $d \in(-0.5,0.5)$ can be extended to a semiparametric class of Gaussian fractionally integrated processes with memory parameter $d \in(-0.5,1.25)$. Since the asymptotic variance of this CLT can be estimated, by data-driven MIR tests for the two cases of stationarity and nonstationarity, so two tests are constructed distinguishing the hypothesis $d<0.5$ and $d \geq 0.5$, as well as a fractional unit roots test distinguishing the case $d=1$ from the case $d<1$. Simulations done on numerous kinds of short-memory, long-memory and non-stationary processes, show both the high accuracy and robustness of this MIR estimator compared to those of usual semiparametric estimators. They also attest of the reasonable efficiency of MIR tests compared to other usual stationarity tests or fractional unit roots tests.

Keywords: Gaussian fractionally integrated processes, semiparametric estimators of the memory parameter, test of long-memory, stationarity test, fractional unit roots test

\section{Introduction}

The set $I(\mathrm{~d})$ of fractionally integrated stochastic process $X=\left(X_{k}\right)_{k \in \mathbb{Z}}$ was defined and used in many articles (see for instance, Granger and Joyeux 1980). Here we consider the following spectral version of this set for $-0.5<d<1.5$ :

\footnotetext{
*Corresponding author: Jean-Marc Bardet, SAMM, Université Panthéon-Sorbonne (Paris I), 90 rue de Tolbiac, 75013 Paris, France, E-mail: bardet@univ-parisi.fr

Béchir Dola, SAMM, Université Panthéon-Sorbonne (Paris I), 90 rue de Tolbiac, 75013 Paris, France, E-mail: bechir.dola@univ-parisi.fr
} 
Set $I(d): X=\left(X_{t}\right)_{t \in \mathbb{Z}}$ is a stochastic process and there exists a continuous function $f^{\star}:[-\pi, \pi] \rightarrow[0, \infty[$ satisfying:

1. if $-0.5<\mathrm{d}<0.5, \mathrm{X}$ is a stationary process with a spectral density $f$ satisfying $f(\lambda)=|\lambda|^{-2 d} f^{\star}(\lambda)$ for all $\lambda \in(-\pi, 0) \cup(0, \pi)$, with $f^{\star}(0)>0$.

2. if $0.5 \leq d<1.5, U=\left(U_{t}\right)_{t \in \mathbb{Z}}=\left(X_{t}-X_{t-1}\right)_{t \in \mathbb{Z}}$ is a stationary process with a spectral density $f$ satisfying $f(\lambda)=|\lambda|^{2-2 d} f^{\star}(\lambda)$ for all $\lambda \in(-\pi, 0) \cup(0, \pi)$, with $f^{\star}(0)>0$.

The case $d \in(0,0.5)$ is the case of long-memory processes, while $-0.5<d \leq 0$ corresponds to short-memory processes while $0.5 \leq d<1.5$ corresponds to nonstationary processes having stationary increments. $\operatorname{ARFIMA}(p, d, q)$ processes (which are linear processes), as well fractional Gaussian noises (with parameter $H=d+1 / 2 \in(0,1)$ ) or fractional Brownian motions (with parameter $H=d-1 / 2 \in$ $(0,1))$ are famous examples of processes satisfying Assumption $I(d)$. The purpose of this paper is twofold: firstly, we establish the consistency of an adaptive datadriven semiparametric estimator of $d$ for any $d \in(-0.5,1.25)$. Secondly, we use this estimator to build new stationarity and fractional unit roots semiparametric tests.

Numerous articles have been devoted to the estimation of $d$ in the case $d \in$ $(-0.5,0.5)$ only. The books of Beran (1994) and Doukhan et al. (2003) provide large surveys of such parametric estimators (as maximum likelihood or Whittle estimators) or semiparametric estimators (as local Whittle, log-periodogram or wavelet based estimators). Here we will focus on the case of semiparametric estimators of processes satisfying Assumption $I(d)$. Even if first versions of local Whittle, log-periodogram and wavelet based estimators are considered in the case $d<0.5$ only (see for instance Robinson 1995a and 1995b; Veitch et al. 2003), new extensions have been provided to estimate $d$ when $d \geq 0.5$ also (see for instance Hurvich and Ray 1995; Velasco 1999a; Velasco and Robinson 2000; Moulines and Soulier 2003; Shimotsu and Phillips 2005; Giraitis et al. 2003, 2006; Abadir et al. 2007 or Moulines et al. 2007). Moreover, adaptive data-driven versions of these estimators have been defined to avoid any trimming or bandwidth parameters, generally required by these methods (see for instance Giraitis et al. 2000; Moulines and Soulier 2003; Veitch et al. 2003 or Bardet and Bibi 2012). The first objective of this paper is to propose for the first time an adaptive data-driven estimator of $d$ satisfying a CLT, providing confidence intervals or tests, that is valid for $d<0.5$ but also for $d \geq 0.5$. This objective is achieved by using Multidimensional Increment Ratio (MIR) statistics.

The original version of the Increment Ratio (IR) statistic was defined in Surgailis et al. (2008) from an observed trajectory $\left(X_{1}, \ldots, X_{N}\right)$ of a process $X$ satisfying $I(d)$ and for any $\ell \in \mathbb{N}^{\star}$ as: 


$$
I R_{N}(\ell):=\frac{1}{N-3 \ell} \sum_{k=0}^{N-3 \ell-1} \frac{\left|\sum_{t=k+1}^{k+\ell} X_{t+\ell}-\sum_{t=k+1}^{k+\ell} X_{t}+\sum_{t=k+\ell+1}^{k+2 \ell} X_{t+\ell}-\sum_{t=k+\ell+1}^{k+2 \ell} X_{t}\right|}{\left|\sum_{t=k+1}^{k+\ell} X_{t+\ell}-\sum_{t=k+1}^{k+\ell} X_{t}\right|+\left|\sum_{t=k+\ell+1}^{k+2 \ell} X_{t+\ell}-\sum_{t=k+\ell+1}^{k+2 \ell} X_{t}\right|} \cdot
$$

Under conditions on $X$, if $\ell \rightarrow \infty$, and $N / \ell \rightarrow \infty$, it is proved that the statistics $I R_{N}(\ell)$ converges to a deterministic monotone function $\Lambda_{0}(d)$ on $(-0.5,1.5)$ and a CLT is also established for $d \in(-0.5,0.5) \cup(0.5,1.25)$ when $\ell$ is large enough with respect to $N$. As a consequence of this CLT and using the Delta-method, the estimator $\widehat{d}_{N}(\ell)=\Lambda_{0}^{-1}\left(I R_{N}(\ell)\right)$, where $d \mapsto \Lambda_{0}(d)$ is a smooth and increasing function defined in eq. [2.6], is a consistent estimator of $d$ satisfying also a CLT (see more details below). However this new estimator was not totally satisfying. Firstly, it requires the knowledge of the second order behavior of the spectral density, which is clearly unknown in practice, to select $\ell$. Secondly, its numerical accuracy is reasonable but clearly lower than those of local Whittle or log-periodogram estimators. As a consequence, in Bardet and Dola (2012), we built a data-driven Multidimensional IR (MIR) estimator $\widetilde{d}_{N}^{(M I R)}$ computed from $\left(\widehat{d}_{N}\left(\ell_{1}\right), \ldots, \widehat{d}_{N}\left(\ell_{p}\right)\right)$ (see its precise definition in eq. [3.2]) improving both these points but only for $-0.5<d<0.5$. This is an adaptive data-driven semiparametric estimator of $d$ achieving the minimax convergence rate (up to a multiplicative logarithm factor) and requiring no regulation of any auxiliary parameter (as bandwidth or trimming parameters). Moreover, its numerical performances are comparable to the ones of local Whittle, log-periodogram or wavelet based estimators.

Here we extend this previous work to the case $0.5 \leq d<1.25$. Hence we obtain a CLT satisfied by $\widetilde{d}_{N}^{(M I R)}$ for all $d \in(-0.5,1.25)$ with an explicit asymptotic variance depending on $d$ only. This especially allows to obtain confidence intervals of $d$ using Slutsky Lemma. The case $d=0.5$ is now studied and this offers new perspectives: our data-driven estimator can be used for building a stationarity (or non-stationarity) test since 0.5 is the "border number" between stationarity and non-stationarity. The case $d=1$ is also now studied and it provides another application of $\widetilde{d}_{N}^{(M I R)}$ to test fractional unit roots, that is to decide between $d=1$ and $d<1$.

There exist several famous stationarity (or non-stationarity) tests. We may cite parametric tests defined by Elliott et al. (1996) or Ng and Perron (1996, 2001). For non parametric stationarity tests we may cite the LMC test (see Leybourne and McCabe, 1994) and the KPSS (Kwiatkowski, Phillips, Schmidt, Shin) test (see Kwiatkowski et al. 1992), improved by the V/S test (see Giraitis et al. 2003). For 
non-stationarity tests we may cite the Augmented Dickey-Fuller test (see Said and Dickey 1984) and the Phillips and Perron test (PP test in the sequel, see Phillips and Perron 1988). All these tests are unit roots tests (except the V/S test which is also a short-memory test), which are, roughly speaking, tests based on the model $X_{t}=\rho X_{t-1}+\varepsilon_{t}$ with $|\rho| \leq 1$. A right-tailed test $d \geq 0.5$ for a process satisfying Assumption $I(\mathrm{~d})$ is therefore a refinement of a basic unit roots test since the case $\rho=1$ is a particular case of $I(1)$ and the case $|\rho|<1$ a particular case of $I(0)$. Thus, a stationarity (or non-stationarity test) based on the estimator of $d$ provides a useful complementary test to usual unit roots tests.

This principle of stationarity test linked to $d$ has been already investigated in many articles. We can cite Robinson (1994), Tanaka (1999), Ling and Li (2001), Ling (2003) or Nielsen (2004). It also was used to define fractional unit roots tests, like the Fractional Dickey-Fuller test defined by Dolado et al. (2002) or the cointegration rank test defined by Breitung and Hassler (2002). However, all these papers provide parametric tests, with a specified model (for instance ARFIMA or ARFIMA-GARCH processes). Extensions proposed by Lobato and Velasco (2007) and Dolado et al. (2008) allow to extend these tests to $I(d)$ processes with ARMA component but requiring the knowledge of the order of this component. Several papers have been recently devoted to the construction of semiparametric tests, see for instance Giraitis et al. (2006), Abadir et al. (2007) or Surgailis et al. (2008). But these semiparametric tests require the knowledge of the second-order expansion of the spectral density at the zero frequency for adjusting a trimming or a bandwidth parameter; an a priori choice of this parameter always implies a bias of the estimator and therefore of the test when this asymptotic expansion is not smooth enough.

The MIR estimator $\widetilde{d}_{N}^{(M I R)}$ does not present this drawback. It converges to d following a CLT with minimax convergence rate without any a priori choice of a parameter. This result is established for time series belonging to the Gaussian semiparametric class $I G(d, \beta)$ defined below (see the beginning of Section 2 ) which is a restriction of the general set $I(d)$. As a consequence, we construct a stationarity test $\tilde{S}_{N}$ which accepts the stationarity assumption when $\widetilde{d}_{N}^{(M I R)} \leq 0.5+s$ with $s$ a threshold only depending on the type I error test, $\widetilde{d}_{N}^{(M I R)}$ and $N$. A nonstationarity test $\tilde{T}_{N}$ accepting the non-stationarity assumption when $\widetilde{d}_{N}^{(M I R)} \leq 0.5-s$ is also proposed. By the same principle, $\widetilde{d}_{N}^{(M I R)}$ also provides a fractional unit roots test $\tilde{F}_{N}$ for deciding between $d=1$ and $d<1$, i. e. whether $\tilde{F}_{N} \geq 1-s^{\prime}$ or not, where $s^{\prime}$ is a threshold depending on the type I error test.

In Section 5, numerous simulations are realized on several models of time series (short and long-memory processes). First, the new MIR estimator $\widetilde{d}_{N}^{(M I R)}$ is 
compared to the most efficient and famous semiparametric estimators for several values of $d \in(-0.5,1.25)$. The performances of $\widetilde{d}_{N}^{(M I R)}$ are convincing: this estimator is accurate and robust for all the considered processes and is globally as efficient as local Whittle, log-periodogram or wavelet based estimators. Secondly, the new stationarity $\tilde{S}_{N}$ and non-stationarity $\tilde{T}_{N}$ tests are compared to the most famous unit roots tests (KPSS, V/S, ADF and PP tests) for numerous $I(d)$ processes. And the results are quite surprising: even on $\operatorname{AR}(1)$ or $\operatorname{ARIMA}(1,1,0)$ processes, $\tilde{S}_{N}$ and $\tilde{T}_{N}$ tests provide convincing results which are comparable to those obtained with ADF and PP tests while those tests are especially built for these specific processes. For long-memory processes (such as ARFIMA processes), the results are clear: $\tilde{S}_{N}$ and $\tilde{T}_{N}$ tests are accurate tests of (non)stationarity while ADF and PP tests are only helpful when $\mathrm{d}$ is close to 0 or 1 . Concerning the new MIR fractional unit roots test $\tilde{F}_{N}$, it provides satisfying results for all considered processes, while fractional unit roots tests such as the fractional Dickey-Fuller test developed by Dolado et al. (2002) or the efficient Wald test introduced by Lobato and Velasco (2007) are respectively only performing for $\operatorname{ARFIMA}(0, d, 0)$ processes or a class of longmemory processes containing $\operatorname{ARFIMA}(p, d, 0)$ processes but not $\operatorname{ARFIMA}(p, d, q)$ processes with $q \geq 1$.

The forthcoming Section 2 is devoted to the definition and asymptotic behavior of MIR estimators of $d$ and Section 3 studies an adaptive MIR estimator. The stationarity and non-stationarity tests are presented in Section 4 while Section 5 deals with the results of simulations, Section 6 provides conclusive remarks and Section 7 contains all the proofs.

\section{The Multidimensional Increment Ratio Statistic}

Now we consider a semiparametric class $\operatorname{IG}(d, \beta)$ which is a refinement of the general class $I(d)$. For $-0.5<d<1.5$ and $\beta>0$ define:

Assumption: $I G(d, \beta): X=\left(X_{t}\right)_{t \in \mathbb{Z}}$ is a Gaussian process such that there exist $\epsilon>0, c_{0}>0, c_{0}^{\prime}>0$ and $c_{1} \in \mathbb{R}$ satisfying:

1. if $d<0.5, X$ is a stationary process with a spectral density $f$ satisfying for all $\lambda \in(-\pi, 0) \cup(0, \pi)$

$$
f(\lambda)=c_{0}|\lambda|^{-2 d}+c_{1}|\lambda|^{-2 d+\beta}+O\left(|\lambda|^{-2 d+\beta+\epsilon}\right) \text { and }\left|f^{\prime}(\lambda)\right| \leq c_{0}^{\prime} \lambda^{-2 d-1} .
$$

2. if $0.5 \leq d<1.5, U=\left(U_{t}\right)_{t \in \mathbb{Z}}=\left(X_{t}-X_{t-1}\right)_{t \in \mathbb{Z}}$ is a stationary process with a spectral density $f$ satisfying for all $\lambda \in(-\pi, 0) \cup(0, \pi)$

$$
f(\lambda)=c_{0}|\lambda|^{2-2 d}+c_{1}|\lambda|^{2-2 d+\beta}+O\left(|\lambda|^{2-2 d+\beta+\epsilon}\right) \text { and }\left|f^{\prime}(\lambda)\right| \leq c^{\prime}{ }_{0} \lambda^{-2 d+1} \text {. }
$$


Note that Assumption $I G(d, \beta)$ is a particular (but still general) case of the set $I(d)$ defined above.

\section{Remark 1:}

- The extension of the definition from $d \in(-0.5,0.5)$ to $d \in[0.5,1.5)$ is classical since the conditions on the process are replaced by conditions on the process' increments.

- The condition on the derivative f' is not really usual. However, this is not a very restrictive condition since it is satisfied by all the classical long-range dependent processes.

- In the literature, all the theoretical results concerning the IR statistic for time series have been obtained under Gaussian assumptions. In Surgailis et al. (2008) and Bardet and Dola (2012), simulations exhibited, that the obtained limit theorems should be also valid for linear processes. However a theoretical proof of such result would require limit theorems for functionals of multidimensional linear processes difficult to be established, even if numerical experiments seem to show that this assumption could be replaced by the assumption that $X$ is a linear process hawing a fourth-moment order like it was done in Giraitis and Surgailis (1990).

In this section, under Assumption $I G(d, \beta)$, we establish central limit theorems which extend to the case $d \in[0.5,1.25)$ those already obtained in Bardet and Dola (2012) for $d \in\left(-0.5\right.$, 0.5). Let $X=\left(X_{k}\right)_{k \in \mathbb{N}}$ be a process satisfying Assumption $I G(d, \beta)$ and $\left(X_{1}, \ldots, X_{N}\right)$ be a path of $X$. The statistic $I R_{N}$ (see its definition in eq. [1.1]) was first defined in Surgailis et al. (2008) as a way to estimate the memory parameter. In Bardet and Surgailis (2011) a simple version of IR-statistic was also introduced to measure the roughness of continuous time processes, and its connection with level crossing index by geometrical arguments. The main interest of such a statistic is to be very robust to additional or multiplicative trends.

As in Bardet and Dola (2012), let $m_{j}=j m, j=1, \ldots, p$ with $p \in \mathbb{N}^{\star}$ and $m \in \mathbb{N}^{\star}$, and define the random vector $\left(\operatorname{IR}_{N}\left(m_{j}\right)\right)_{1 \leq j \leq p}$. In the sequel we naturally extend the results obtained for $m \in \mathbb{N}^{\star}$ to $m \in(0, \infty)$ by the convention: $\left(I_{N}(j m)\right)_{1 \leq j \leq p}=\left(I R_{N}\right.$ $(j[m]))_{1} \leq_{j} \leq_{p}$ (which does not change the asymptotic results).

For $H \in(0,1)$, let $B_{H}=\left(B_{H}(t)\right)_{\mathrm{t} \in \mathbb{R}}$ be a standard fractional Brownian motion, i.e. a centered Gaussian process having stationary increments and such as $\operatorname{Cov}\left(B_{H}(t)\right.$, $\left.B_{H}(s)\right)=\frac{1}{2}\left(|t|^{2 H}+|s|^{2 H}-|t-s|^{2 H}\right)$. Now, using obvious modifications of Surgailis et al. (2008), for $d \in(-0.5,1.25)$ and $p \in \mathbb{N}^{\star}$, define the stationary multidimensional centered Gaussian processes $\left(Z_{d}^{(1)}(\tau), \ldots, Z_{d}^{(p)}(\tau)\right)$ such as for $\tau \in \mathbb{R}$, 
$Z_{d}^{(j)}(\tau):= \begin{cases}\frac{\sqrt{2 d(2 d+1)}}{\sqrt{\left|4^{d+0.5}-4\right|}} \int_{0}^{1}\left(B_{d-0.5}(\tau+s+j)-B_{d-0.5}(\tau+s)\right) d s & \text { if } d \in(0.5,1.25) \\ \frac{1}{\sqrt{\left|4^{d+0.5}-4\right|}}\left(B_{d+0.5}(\tau+2 j)-2 B_{d+0.5}(\tau+j)+B_{d+0.5}(\tau)\right) & \text { if } d \in(-0.5,0.5) .\end{cases}$

Using a continuous extension when $d \rightarrow 0.5$ of the covariance of $Z_{d}^{(j)}(\tau)$, we also define the stationary multidimensional centered Gaussian processes $\left(Z_{0.5}^{(1)}\right.$ $\left.(\tau), \ldots, Z_{0.5}^{(p)}(\tau)\right)$ with covariance such as:

$\operatorname{Cov}\left(Z_{0.5}^{(i)}(0), Z_{0.5}^{(j)}(\tau)\right):=\frac{1}{4 \log 2}(-h(\tau+i-j)+h(\tau+i)+h(\tau-j)-h(\tau))$ for $\tau \in \mathbb{R}$,

where $h(x)=\frac{1}{2}\left(|x-1|^{2} \log |x-1|+|x+1|^{2} \log |x+1|-2|x|^{2} \log |x|\right)$ for $x \in \mathbb{R}$, using the convention $0 \times \log 0=0$. Now, we establish a multidimensional CLT satisfied by $\left(I R_{N}(j m)\right)_{1 \leq j \leq p}$ for all $d \in(-0.5,1.25)$ :

Proposition 1: Assume that Assumption IG(d, $\beta$ ) holds with $-0.5 \leq d<1.25$ and $\beta>0$. Then

$$
\sqrt{\frac{N}{m}}\left(I R_{N}(j m)-\mathrm{E}\left[I R_{N}(j m)\right]\right)_{i \leq j \leq p} \underset{[N / m] \wedge m \rightarrow \infty}{\stackrel{\mathcal{L}}{\longrightarrow}} \mathcal{N}\left(0, \Gamma_{p}(d)\right)
$$

with $\Gamma_{p}(d)=\left(\sigma_{i, j}(d)\right)_{1 \leq i, j \leq p}$ where for $i, j \in\{1, \ldots, p\}$,

$$
\sigma_{i, j}(d):=\int_{-\infty}^{\infty} \operatorname{Cov}\left(\frac{\left|Z_{d}^{(i)}(0)+Z_{d}^{(i)}(i)\right|}{\left|Z_{d}^{(i)}(0)\right|+\left|Z_{d}^{(i)}(i)\right|}, \frac{\left|Z_{d}^{(j)}(\tau)+Z_{d}^{(j)}(\tau+j)\right|}{\left|Z_{d}^{(j)}(\tau)\right|+\left|Z_{d}^{(j)}(\tau+j)\right|}\right) d \tau .
$$

The proof of this proposition as well as all the other proofs can be found in Section 7.

In the sequel, we will assume that $\Gamma_{p}(d)$ is a positive definite matrix for all $d \in(-0.5,1.25)$. Extensive numerical experiments seem to give strong evidence of such a property. Now, the CLT eq. [2.4] can be used for estimating $d$. To begin with,

Property 1: Let $X$ satisfy Assumption $I G(d, \beta)$ with $0.5 \leq d<1.5$ and $0<\beta \leq 2$. Then, there exists a non-vanishing constant $K(d, \beta)$ depending only on $d$ and $\beta$ such that for $m$ large enough,

$$
\mathrm{E}\left[I R_{N}(m)\right]= \begin{cases}\Lambda_{0}(d)+K(d, \beta) \times m^{-\beta}(1+o(1)) & \text { if } \beta<1+2 d \\ \Lambda_{0}(d)+K(0.5, \beta) \times m^{-2} \log m(1+o(1)) & \text { if } \beta=2 \text { and } d=0.5\end{cases}
$$


with $\Lambda_{0}(d):=\Lambda(\rho(d))$ where $\rho(d):= \begin{cases}\frac{4^{d+1.5}-9^{d+0.5}-7}{2\left(4-4^{d+0.5}\right)} & \text { for } \quad 0.5<d<1.5 \\ \frac{9 \log (3)}{8 \log (2)}-2 & \text { for } \quad d=0.5\end{cases}$

and $\Lambda(r):=\frac{2}{\pi} \arctan \sqrt{\frac{1+r}{1-r}}+\frac{1}{\pi} \sqrt{\frac{1+r}{1-r}} \log \left(\frac{2}{1+r}\right) \quad$ for $|r| \leq 1$.

Therefore by choosing $m$ and $N$ such as $(\sqrt{N / m}) m^{-\beta} \log m \rightarrow 0$ when $m, N \rightarrow \infty$, the term $E[I R(j m)]$ can be replaced by $\Lambda_{0}(d)$ in Proposition 1 . Then, using the Delta-method with the function $\left(x_{i}\right)_{1 \leq i \leq p} \mapsto\left(\Lambda_{0}^{-1}\left(x_{i}\right)\right)_{1 \leq i \leq p}$ (the function $d \in(-0.5,1.5) \rightarrow \Lambda_{0}(d)$ is a $\mathcal{C}^{\infty}$ increasing function), we obtain:

Theorem 1: Let $\widehat{d}_{N}(j m):=\Lambda_{0}^{-1}\left(I R_{N}(j m)\right)$ for $1 \leq j \leq p$. Assume that Assumption $I G(d, \beta)$ holds with $0.5 \leq d<1.25$ and $0<\beta \leq 2$. Then if $m \sim C N^{\alpha}$ with $C>0$ and $(1+2 \beta)^{-1}<\alpha<1$,

$$
\sqrt{\frac{N}{m}}\left(\widehat{d}_{N}(j m)-d\right)_{1 \leq j \leq p} \underset{N \rightarrow \infty}{\stackrel{\mathcal{L}}{\longrightarrow}} \mathcal{N}\left(0,\left(\Lambda_{0}^{\prime}(d)\right)^{-2} \Gamma_{p}(d)\right) .
$$

This result is an extension to the case $0.5 \leq d \leq 1.25$ from the case $-0.5<d<0.5$ already obtained in Bardet and Dola (2012). Note that the consistency of $\widehat{d}_{N}(j m)$ is ensured when $1.25 \leq d<1.5$ but the previous CLT does not hold (the asymptotic variance of $\sqrt{\frac{N}{m}} \widehat{d}_{N}(j m)$ diverges to $\infty$ when $d>1.25$, see Surgailis et al. 2008).

Now define

$$
\widehat{\Sigma}_{N}(m):=\left(\Lambda_{0}^{\prime}\left(\widehat{d}_{N}(m)\right)^{-2} \Gamma_{p}\left(\widehat{d}_{N}(m)\right)\right) .
$$

The function $d \in(-0.5,1.5) \mapsto \sigma(d) / \Lambda^{\prime}(d)$ is $C^{\infty}$ and therefore, under assumptions of Theorem 1,

$$
\widehat{\Sigma}_{N}(m) \underset{N \rightarrow \infty}{\stackrel{p}{\longrightarrow}}\left(\Lambda_{0}^{\prime}(d)\right)^{-2} \Gamma_{p}(d) .
$$

Thus, a pseudo-generalized least square estimation (PGLSE) of $d$ can be defined by

$$
\widetilde{d}_{N}(m):=\left(J_{p}^{\mathrm{T}}\left(\widehat{\Sigma}_{N}(m)\right)^{-1} J_{p}\right)^{-1} J_{p}^{\mathrm{T}}\left(\widehat{\Sigma}_{N}(m)\right)^{-1}\left(\widehat{d}_{N}\left(m_{i}\right)\right)_{1 \leq i \leq p}
$$

with $J_{p}:=(1)_{1} \leq_{j} \leq_{p}$ and denoting $J_{p}^{\mathrm{T}}$ its transpose. From a Gauss-Markov Theorem type (see again Bardet and Dola 2012), the asymptotic variance of $\widetilde{d}_{N}(m)$ is smaller than the one of any $\widehat{d}_{N}(j m), j=1, \ldots, p$. Hence, we obtain under the assumptions of Theorem 1:

$$
\sqrt{\frac{N}{m}}\left(\widetilde{d}_{N}(m)-d\right) \underset{N \rightarrow \infty}{\stackrel{L}{\longrightarrow}} \mathcal{N}\left(0, \Lambda_{0}^{\prime}(d)^{-2}\left(J_{p}^{\mathrm{T}} \Gamma_{p}^{-1}(d) J_{p}\right)^{-1}\right) .
$$




\section{The Adaptive Data-Driven Version of the Estimator}

Theorem 1 and CLT eq. [2.10] require the knowledge of $\beta$ to be applied. But in practice $\beta$ is unknown. The procedure defined in Bardet and Bibi (2012) or Bardet and Dola (2012) can be used for obtaining a data-driven selection of an optimal sequence $\left(\tilde{m}_{N}\right)$ derived from an estimation of $\beta$. Since the case $d \in(-0.5,0.5)$ was studied in Bardet and Dola (2012) we consider here $d \in[0.5,1.25)$ and for $\alpha \in(0,1)$, define

$Q_{N}(\alpha, d):=\left(\widehat{d}_{N}\left(j N^{\alpha}\right)-\widetilde{d}_{N}\left(N^{\alpha}\right)\right)_{1 \leq j \leq p}^{\mathrm{T}}\left(\widehat{\Sigma}_{N}\left(N^{\alpha}\right)\right)^{-1}\left(\widehat{d}_{N}\left(j N^{\alpha}\right)-\left(\widetilde{d}_{N}\left(N^{\alpha}\right)\right)\right)_{1 \leq j \leq p}$,

which corresponds to the sum of the pseudo-generalized squared distance between the points $\left(\widehat{d}_{N}\left(j N^{\alpha}\right)\right)_{j}$ and PGLSE of $d$. Note that by the previous convention, $\widehat{d}_{N}\left(j N^{\alpha}\right)=\widehat{d}_{N}\left(j\left[N^{\alpha}\right]\right)$ and $\widetilde{d}_{N}\left(N^{\alpha}\right)=\widetilde{d}_{N}\left(\left[N^{\alpha}\right]\right)$. Then $\widehat{Q}_{N}(\alpha)$ can be minimized on a discretization of $(0,1)$ and define:

$$
\widehat{\alpha}_{N}:=\operatorname{Argmin}_{\alpha \in \mathcal{A}_{N}} \widehat{Q}_{N}(\alpha) \text { with } \mathcal{A}_{N}=\left\{\frac{2}{\log N}, \frac{3}{\log N}, \ldots, \frac{\log [N / p]}{\log N}\right\} .
$$

Remark 2: The choice of the set of discretization $\mathcal{A}_{N}$ is implied by our proof of convergence of $\hat{\alpha}_{N}$. If the interval $(0,1)$ is stepped in $N^{c}$ points, with $c>0$, the used proof cannot attest this convergence. However log $N$ may be replaced in the previous expression of $\mathcal{A}_{N}$ by any negligible function of $N$ compared to functions $N^{c}$ with $c>0$ (for instance, $(\log N)^{a}$ or a $\log N$ with $\left.a>0\right)$.

From the central limit theorem eq. [2.8] one deduces the following limit theorem:

Proposition 2: Assume that Assumption $\operatorname{IG}(d, \beta)$ holds with $0.5 \leq d<1.25$ and $0<\beta \leq 2$. Then,

$$
\widehat{\alpha}_{N} \underset{N \rightarrow \infty}{\stackrel{P}{\longrightarrow}} \alpha^{\star}=\frac{1}{(1+2 \beta)} .
$$

Finally define

$$
\tilde{m}_{N}:=N^{\tilde{\alpha}_{N}} \text { with } \tilde{a}_{N}:=\widehat{a}_{N}+\frac{6 \widehat{a}_{N}}{(p-2)\left(1-\widehat{a}_{N}\right)} \cdot \frac{\log \log N}{\log N} .
$$

and the estimator 


$$
\widetilde{d}_{N}^{(M I R)}:=\widetilde{d}_{N}\left(\tilde{m}_{N}\right)=\widetilde{d}_{N}\left({\widetilde{\alpha^{\alpha}}}\right) .
$$

(the definition and use of $\tilde{\alpha}_{N}$ instead of $\hat{\alpha}_{N}$ are explained just before Theorem 2 in Bardet and Dola 2012). The following theorem provides the asymptotic behavior of the estimator $\widetilde{d}_{N}^{(M I R)}$ :

Theorem 2: Under assumptions of Proposition 2,

$$
\sqrt{\frac{N}{N^{\tilde{\alpha}_{N}}}}\left(\widetilde{d}_{N}^{(M I R)}-d\right) \underset{N \rightarrow \infty}{\stackrel{\mathcal{L}}{\longrightarrow}} \mathcal{N}\left(0 ; \Lambda_{0}^{\prime}(d)^{-2}\left(J_{p}^{\mathrm{T}} \Gamma_{p}^{-1}(d) J_{p}\right)^{-1}\right) .
$$

Moreover, $\forall \rho>\frac{2(1+3 \beta)}{(p-2) / \beta}, \frac{N^{\frac{\beta}{1+2 \beta}}}{(\log N)^{\rho}} .\left|\widetilde{d}_{N}^{(M I R)}-d\right| \underset{N \rightarrow \infty}{\stackrel{P}{\longrightarrow}} 0$.

The convergence rate of $\widetilde{d}_{N}^{(M I R)}$ is the same (up to a multiplicative logarithm factor) than the one of minimax estimator of $d$ in this semiparametric framework (see Giraitis et al. 1997). As it was already established in Surgailis et al. (2008), the use of IR statistics confers a robustness of $\widetilde{d}_{N}^{(M I R)}$ to smooth additive or multiplicative trends (see also the results of simulations thereafter). The additional advantage of $\widetilde{d}_{N}^{(M I R)}$ with respect to other adaptive estimators of $d$ (see Moulines and Soulier 2003, for an overview over frequency domain estimators of d) is the central limit theorem eq. [3.3] satisfied by $\widetilde{d}_{N}^{(M I R)}$. This central limit theorem provides asymptotic confidence intervals on $d$ which are unobtainable for instance with FEXP or local periodogram adaptive estimator (see respectively Iouditsky et al. 2001, and Giraitis et al. 2000 or Henry 2007). Moreover $\widetilde{d}_{N}^{(M I R)}$ can be used for $d \in(-0.5,1.25)$, i.e. as well for stationary and non-stationary processes, without modifications in its definition. Both these advantages allow to define stationarity and fractional unit roots tests based on $\widetilde{d}_{N}^{(M I R)}$.

\section{Stationarity, Non-Stationarity and Fractional Unit Roots Tests}

Assume that $\left(X_{1}, \ldots, X_{N}\right)$ is an observed trajectory of a process $X=\left(X_{k}\right)_{k \in \mathbb{Z}}$. We define here new stationarity, non-stationarity and fractional unit roots tests for $X$ based on $\widetilde{d}_{N}^{(M I R)}$.

\subsection{A Stationarity Test}

There exist many stationarity and non-stationarity tests. The most famous stationarity tests are certainly the following unit roots tests: 
- $\quad$ The KPSS (Kwiatkowski, Phillips, Schmidt, Shin) test (see Kwiatkowski et al. 1992);

- The V/S test (see its presentation in Giraitis et al. 2001) which was first defined for testing the presence of long-memory versus short-memory. As it was already notified in Giraitis et al. 2003, 2006), the V/S test is also more powerful than the KPSS test for testing the stationarity.

- A test based on unidimensional IR statistic and developed in Surgailis et al. (2008).

More precisely, we consider here the following statistical hypothesis test:

- $\quad$ Hypothesis $H_{0}$ (stationarity): $\left(X_{t}\right)_{t \in \mathbb{Z}}$ is a process satisfying Assumption $I G$ $(d, \beta)$ with $d \in(-0.5,0.5)$ and $0<\beta \leq 2$.

- Hypothesis $H_{1}$ (non-stationarity): $\left(X_{t}\right)_{t \in \mathbb{Z}}$ is a process satisfying Assumption $\operatorname{IG}(d, \beta)$ with $d \in[0.5,1.25)$ and $0<\beta \leq 2$.

We use a test based on $\widetilde{d}_{N}^{(M I R)}$ for deciding between both these hypothesis. Hence from the previous CLT eq. [3.3] and with a significance level $\alpha$, define

$$
\tilde{S}_{N}:=1 \widetilde{d}_{N}^{(M I R)}>0.5+\sigma_{p}(0.5) q_{1}-\alpha N^{\left(\tilde{\alpha} N^{-1}\right) / 2},
$$

where $\sigma_{p}(0.5)=\left(\Lambda^{\prime}{ }_{0}(0.5)^{-2}\left(J_{p}^{\mathrm{T}} \Gamma_{p}^{-1}(0.5) J_{p}\right)^{-1}\right)^{1 / 2}$ (see eq. [3.3] and $q_{1-\alpha}$ is the $(1-\alpha)$ quantile of a standard Gausian random variable $N(0,1)$.

Then we define the following rules of decision:

" $H_{0}$ (stationarity) is accepted when $\tilde{S}_{N}=0$ and rejected when $\tilde{S}_{N}=1$. ,

Remark 3: In fact, the previous stationarity test $\tilde{S}_{N}$ defined in eq. [4.1] can also be seen as a semiparametric test $d<d_{0}$ versus $d \geq d_{0}$ with $d_{0}=0.5$. It is obviously possible to extend it to any value $d_{0} \in(-0.5,1.25)$ by defining $\tilde{S}_{N}^{\left(d_{0}\right)}:=1 \widetilde{d}_{N}^{(M I R)}>d_{0}+\sigma_{p}\left(d_{0}\right) q_{1-\alpha} N^{(\tilde{\alpha} N-1) / 2}$. The particular case $d_{0}=1$ will be considered thereafter as a fractional unit roots test.

From previous results, it is clear that:

Property 2: Under Hypothesis $H_{0}$, the asymptotic type I error of the test $\tilde{S}_{N}$ is $\alpha$ and under Hypothesis $H_{1}$, the test power tends to 1.

Moreover, this test can be used as a unit roots (UR) test. Indeed, define the following typical problem of UR test. Let $X_{t}=a t+b+\varepsilon_{t}$, with $(a, b) \in \mathbb{R}^{2}$, and $\varepsilon_{t}$ an $\operatorname{ARIMA}(p, d, q)$ with $d=0$ or $d=1$. Then, a (simplified) problem of a UR test is to decide between: 
- $\quad H_{0}^{U R}: \mathrm{d}=0$ and $\left(\varepsilon_{t}\right)$ is a stationary $\operatorname{ARMA}\left(p^{\prime}, q^{\prime}\right)$ process.

- $H_{1}^{U R}: d=1$ and $\left(\varepsilon_{t}-\varepsilon_{t-1}\right)_{t}$ is a stationary $\operatorname{ARMA}\left(p^{\prime}, q^{\prime}\right)$ process.

Then,

Property 3: Under Hypothesis $H_{0}^{U R}$, the type I error of this unit roots test problem using $\tilde{S}_{N}$ decreases to 0 when $N \rightarrow \infty$ and under Hypothesis $H_{1}^{U R}$, the test power tends to 1 .

\subsection{A Non-Stationarity Test}

Unit roots tests are also often used as non-stationarity test. Hence, between the most famous non-stationarity tests and in a nonparametric framework, consider - $\quad$ The Augmented Dickey-Fuller (ADF) test (see Said and Dickey, 1984);

- $\quad$ The Phillips and Perron (PP) test (see for instance Phillips and Perron 1988).

Using the statistic $\widetilde{d}_{N}^{(M I R)}$ we propose a new non-stationarity test $\tilde{T}_{N}$ for deciding between:

- Hypothesis $H_{0}^{\prime}$ (non-stationarity): $\left(X_{t}\right)_{t \in \mathbb{Z}}$ is a process satisfying Assumption $\overline{I G(d, \beta) \text { with } d} \in[0: 5 ; 1: 25)$ and $\beta \in(0,2]$.

- Hypothesis $H_{1}^{\prime}$ (stationarity): $\left(X_{t}\right)_{t \in \mathbb{Z}}$ is a process satisfying Assumption

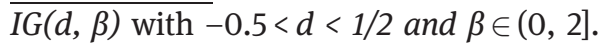

Then, the decision rule of the test under the significance level $\alpha$ is the following:

"Hypothesis $H_{0}^{\prime}$ is accepted when $\tilde{T}_{N}=1$ and rejected when $\tilde{T}_{N}=0$ "

where

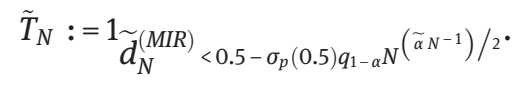

Then,

Property 4: Under Hypothesis $H_{0}^{\prime}$, the asymptotic type I error of the test $\tilde{T}_{N}$ is $\alpha$ and under Hypothesis $H_{1}^{\prime}$ the test power tends to 1.

As previously, this test can also be used as a unit roots test where $X_{t}=a t+b+\varepsilon_{t}$, with $(a, b) \in \mathbb{R}^{2}$, and $\varepsilon_{t}$ an $\operatorname{ARIMA}(p, d, q)$ with $d=0$ or $d=1$. We consider here a "second" simplified problem of unit roots test which is to decide between: 
- $\quad H_{0}^{U R^{\prime}}: d=1$ and $\left(\varepsilon_{t}-\varepsilon_{t-1}\right)$ is a stationary $\operatorname{ARMA}\left(p^{\prime}, q^{\prime}\right)$ process.

- $\quad H_{0}^{U R^{\prime}}: d=0$ and $\left(\varepsilon_{t}\right)_{t}$ is a stationary $\operatorname{ARMA}\left(p^{\prime}, q^{\prime}\right)$ process.

Then,

Property 5: Under Hypothesis $H_{0}^{U R^{\prime}}$, the type I error of the unit roots test problem using $\tilde{T}_{N}$ decreases to 0 when $N \rightarrow \infty$ and under Hypothesis $H_{1}^{U R^{\prime}}$ the test power tends to 1 .

\subsection{A Fractional Unit Roots Test}

Fractional unit roots tests have also been defined for specifying the eventual long-memory property of the process in a unit roots test. In our Gaussian framework, they consist on testing

- Hypothesis $H_{0}^{F U R}:\left(X_{t}\right)_{t \in \mathbb{Z}}$ is a "random walk"-type process such as:

$$
X_{t}=X_{t-1}+u_{t}
$$

with $\left(u_{t}\right)_{t}$ a process satisfying Assumption $I G(0, \beta)$ with $0<\beta \leq 2$. Therefore $\left(X_{t}\right)$ is a process satisfying Assumption $I G(1, \beta)$.

- Hypothesis $H_{1}^{F U R}:\left(X_{t}\right)_{t \in \mathbb{Z}}$ is a process satisfying the following relation:

$$
X_{t}=X_{t-1}+\phi \Delta^{d_{1}} X_{t-1}+u_{t}
$$

where $\left(u_{t}\right)_{t}$ is a process satisfying Assumption $\operatorname{IG}(0, \beta)$ with $0<\beta \leq 2, \phi<0$, and $\Delta^{d_{1}}$ is the fractional integration operator of order $0<d_{1}<1$, i.e. $\Delta^{d_{1}} X_{t-1}=\sum_{i=0}^{t=1} \pi_{i}\left(d_{1}\right) X_{t-1-i}$ and $\pi_{i}\left(d_{1}\right)=\Gamma\left(i-d_{1}\right)\left(\Gamma(i+1) \Gamma\left(-d_{1}\right)\right)^{-1}$.

After computations, it follows that if $X$ satisfies eq. [4.4], then $X$ satisfies Assumption $I G\left(d_{1}, \beta\right)$. There exist several fractional unit roots tests (see for example, Robinson 1994; Tanaka 1999; Dolado et al. 2002; or more recently, Kew and Harris 2009). It is clear that the estimator $\widetilde{d}_{N}^{(M I R)}$ can be used in such a framework for testing fractional unit roots by comparing $\widetilde{d}_{N}^{(M I R)}$ to 1 . Hence, the decision rule of the test under the significance level $\alpha$ is the following:

"Hypothesis $H_{0}^{F U R}$ is accepted when $\tilde{F}_{N}=1$ and rejected when $\tilde{F}_{N}=0$ "

where

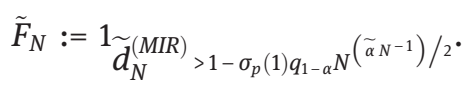

Then as previously 
Property 6: Under Hypothesis $H_{0}^{F U R}$, the asymptotic type I error of the test $\tilde{F}_{N}$ is $\alpha$ and under Hypothesis $H_{1}^{F U R}$ the test power tends to 1.

\section{Results of Simulations}

\subsection{Numerical Procedure for Computing the Estimator and Tests}

First of all, softwares used in this Section are available on http: / / samm. univparis1.fr/-Jean-Marc-Bardet with a free access on (in Matlab language).

The concrete procedure for applying the MIR-test of stationarity is the following:

1. using additional simulations (performed on ARMA, ARFIMA, FGN processes and not presented here in order to avoid overloading the paper), we have observed that the value of the parameter $p$ is not really important with respect to the accuracy of the test (there are less than $10 \%$ of fluctuations on the value of $\widetilde{d}_{N}^{(M I R)}$ when $p$ varies). However, for optimizing our procedure (in the sense of minimizing from simulation the mean square error of the $d$ estimation) we chose $p$ as a stepwise function of $N$ :

$$
p=5 \times 1_{\{N<120\}}+10 \times 1_{\{120 \leq N<800\}}+15 \times 1_{\{800 \leq N<10000\}}+20 \times 1_{\{N \geq 10000\}} .
$$

2. as the values of $\sigma_{p}(0.5)$ and $\sigma_{p}(1)$ are essential for computing the thresholds of the tests, we have estimated them and obtained:

$-\sigma_{5}(0.5) \simeq 0.9082, \sigma_{10}(0.5) \simeq 0.8289, \sigma_{15}(0.5) \simeq 0.8016$ and $\sigma_{20}(0.5) \simeq$ 0.7861 .

- $\quad \sigma_{5}(1) \simeq 0.8381, \sigma_{10}(1) \simeq 0.8102, \sigma_{5}(1) \simeq 0.8082$ and $\sigma_{20}(1) \simeq 0.7929$.

3. then after computing $\tilde{m}_{N}$ presented in Section 3 , the adaptive estimator $\widetilde{d}_{N}^{(M I R)}$ defined in eq. [3.2], the test statistics $\tilde{S}_{N}$ defined in eq. [4.1], $\tilde{T}_{N}$ defined in eq. [4.2] and $\tilde{F}_{N}$ defined in eq. [4.5] are computed.

\subsection{Monte-Carlo Experiments on Several Time Series}

In the sequel the results are obtained from 1,000 generated independent trajectories of each process defined below. The concrete procedures of generation of these processes are obtained from the circulant matrix method, as detailed in Doukhan et al. (2003). The simulations are realized for different values of $d$ and $N$ and processes which satisfy Assumption $\operatorname{IG}(d, \beta)$ : 
1. the usual $\operatorname{ARIMA}\left(p^{\prime}, d, q^{\prime}\right)$ processes with respectively $d=0$ or $d=1$ and an innovation process which is a Gaussian white noise. Such processes satisfy Assumption $\operatorname{IG}(0,2)$ or $I G(1,2)$ (respectively);

2. the $\operatorname{ARFIMA}\left(p^{\prime}, d, q^{\prime}\right)$ processes with parameter $d$ such that $d \in(-0.5,1.25)$ and an innovation process which is a Gaussian white noise. Such ARFIMA $\left(p^{\prime}, d, q^{\prime}\right)$ processes satisfy Assumption $\operatorname{IG}(d, 2)$ (note that ARIMA processes are particular cases of ARFIMA processes).

3. the Gaussian stationary processes $X^{\left(d, c_{1}, d\right)}$ with the spectral density

$$
f_{3}(\lambda)=\frac{1}{|\lambda|^{2 d}}\left(1+c_{1}|\lambda|^{\beta}\right) \quad \text { for } \quad \lambda \in[-\pi, 0) \cup(0, \pi],
$$

with $d \in(-0.5,1.5), c_{1}>0$ and $\beta \in(0, \infty)$. Therefore the spectral density $f_{3}$ implies that Assumption $\operatorname{IG}(d, \beta)$ holds. In the sequel we will first use $c_{1}=1$ and $\beta=0.1$, implying that the second order term of the spectral density is "less negligible" than in case of ARFIMA processes, and $c_{1}=0$, implying that the second order term of the spectral density is "more negligible" than in case of ARFIMA processes.

4. the Gaussian stationary processes $X^{(d, \log )}$, such as its spectral density is

$$
f_{4}(\lambda)=\frac{1}{|\lambda|^{2 d}}(1+|\log (\lambda)||\lambda|) \quad \text { for } \quad \lambda \in[-\pi, 0) \cup(0, \pi],
$$

$d \in(-0.5,1.5)$ Therefore the spectral density $f_{4}$ implies that Assumption $I(d)$ holds, but not $I G(d, \beta)$ stricto sensu.

5. the Gaussian non-stationary process $X_{t}^{(t r e n d)}$ which can be written as $X_{t}^{(\text {trend })}=a_{n}(t)+\sigma_{n}(t) \times A R F I M A(0, d, 0)$, where the additive and multiplicative trends are respectively $a_{n}(t)=\sin (2 \pi t / n)$ and $\sigma_{n}(t)=\sqrt{2 t / n}$ (for us we chose a non-polynomial but smooth additive trend).

\subsubsection{Comparison of $\widetilde{d}_{N}^{(M I R)}$ with other Semiparametric Estimators of $d$}

Here we first compare the performance of the data-driven MIR estimator $\widetilde{d}_{N}^{(M I R)}$ with other famous semiparametric estimators of $d$ :

- $\widehat{d}_{N}^{(I R)}$ is the original version of the IR based estimator defined in Surgailis et al. (2008). As it was recommended in that article, we chose $m=10$.

- $\tilde{d}_{N}^{(M S)}$ is the global log-periodogram estimator introduced by Moulines and Soulier (2003), also called FEXP estimator, with bias-variance balance parameter $K=2$. Such an estimator was shown to be consistent for $d \in(-0.5,1)$. This semiparametric estimator is an adaptive data-driven estimator of $d$.

- $\widetilde{d}_{N}^{(A D G)}$ is the extended local Whittle estimator defined by Abadir et al. (2007) which is consistent for $d>-3 / 2$. It is a generalization of the local Whittle 
estimator introduced by Robinson (1995b), consistent for $d<0.75$, following a first extension proposed by Phillips (1999) and Shimotsu and Phillips (2005). This estimator avoids the tapering used for instance in Velasco (1999b) or Hurvich and Chen (2000). The trimming parameter is chosen as $m=N^{0.65}$ (this is not an adaptive data-driven estimator) following the numerical recommendations of Abadir et al. (2007).

- $\quad \tilde{d}_{N}^{(W A V)}$ is an adaptive data-driven wavelet based estimator introduced in Bardet and Bibi (2012) using a Lemarie-Meyer type wavelet (another similar choice could be the adaptive wavelet estimator introduced in Veitch et al. 2003, using a Daubechie's wavelet, but its robustness properties are slightly less interesting). The asymptotic normality of such estimator is established for $d>-0.5$ (when the number of vanishing moments of the wavelet function is large enough).

Note that only $\widehat{d}_{N}^{(I R)}$ and $\widehat{d}_{N}^{(A D G)}$ are not data-driven adaptive among the 5 estimators. Table 1 provides the results of simulations for $\operatorname{ARIMA}(1, d, 0)(N=500, N=5,000$ and $N=50,000)$. For $\operatorname{ARFIMA}(0, d, 0), \operatorname{ARFIMA}(1, d, 1), X^{(\mathrm{d}, \log )}, X^{(\mathrm{d}, 0,1)}, X^{(\mathrm{d}, \log )}$ and $X^{(\text {trend })}$ processes and several values of $d$, the results of simulations are presented for $N=500$ (Table 2), $N=5000$ (Table 3) and $N=50,000$ (Table 4).

Conclusions of simulations: Even if the estimator $\widehat{d}_{N}^{(A D G)}$ often provides the more accurate estimation of $d$ for stationary processes, it is not more accurate anymore than $\widehat{d}_{N}^{(M I R)}$ in case of trended time series. Moreover since this is not a data-driven estimator, with a bandwidth $m$ fixed to be $N^{0.65}$, it is not a consistent estimator when $\beta$ is small enough: this is such the case for $X^{(d, \log )}$ where we observe that the MSE is globally larger for $N=50,000$ than for $N=5,000$. The estimator $\widehat{d}_{N}^{(M I R)}$ is a very good trade-off with always one of the smallest $\sqrt{M S E}$ among the 5 semiparametric estimators and almost never bad results (except perhaps for $X^{(t r e n d)}, N=500$ and $d<0.5)$. Moreover, the larger $N$ the more efficient $\widehat{d}_{N}^{(M I R)}$ with respect to the other estimators. Note also that the use of a data-driven multidimensional version of $I R$ statistics (i.e. the estimator $\widehat{d}_{N}^{(M I R)}$ ) considerably improves the quality of the estimation with respect to the original estimator based on unidimensional IR statistics (the estimator $\widehat{d}_{N}^{(I R)}$ ). Finally the other data-driven estimators $d^{(M S)}$ and $d^{(W A V)}$ provide correct results but they are often less efficient than $\widehat{d}_{N}^{(M I R)}$.

\subsubsection{Comparison of MIR Tests $\tilde{S}_{N}$ and $\tilde{T}_{N}$ with other Stationarity or Non-Stationarity Tests}

Monte-Carlo experiments were done for evaluating the performances of new tests $\tilde{S}_{N}$ and $\tilde{T}_{N}$ and for comparing them to most famous stationarity tests 


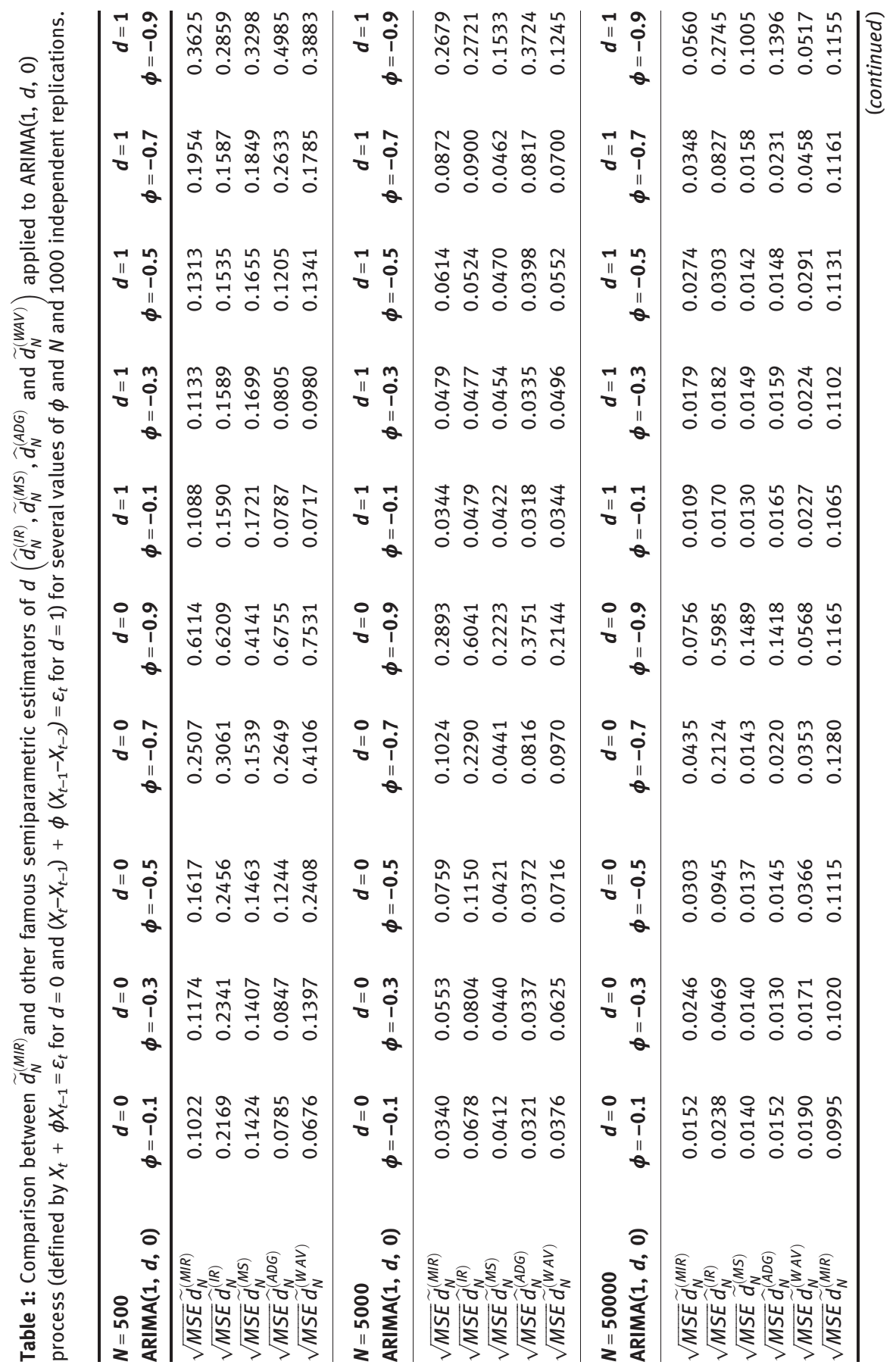




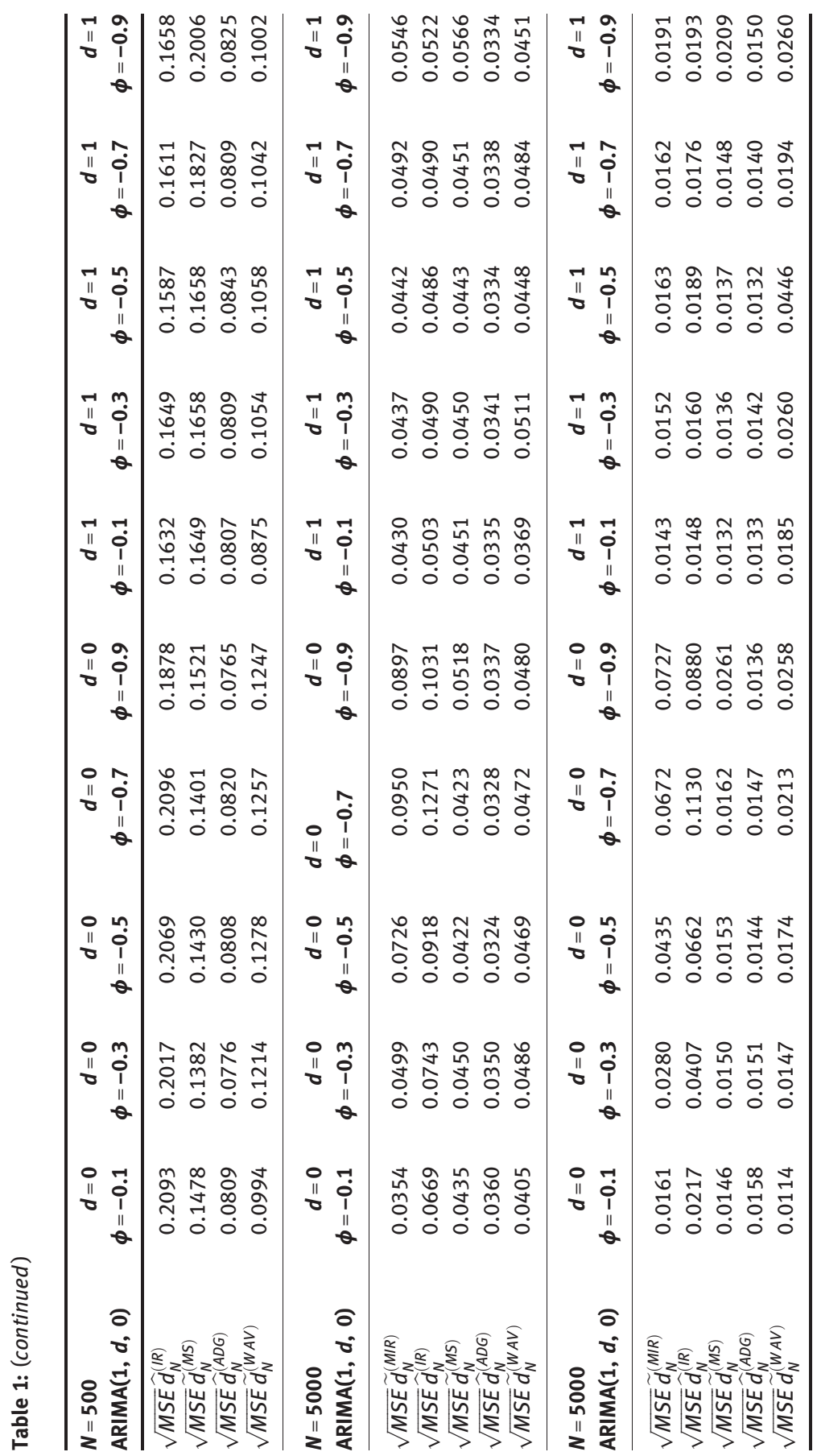


Table 2: Comparison between $\widetilde{d}_{N}^{(M I R)}$ and other famous semiparametric estimators of $d\left(\widetilde{d}_{N}^{(I R)}\right.$, $\widetilde{d}_{N}^{(M S)}, \widehat{d}_{N}^{(A D G)}$ and $\left.\widetilde{d}_{N}^{(W A V)}\right)$ applied to fractionally integrated processes for $N=500$, several values of $d \in(-0.5,1.25)$ and 1000 independent replications.

\begin{tabular}{|c|c|c|c|c|c|c|c|c|}
\hline$N=500$ & $=-0.2$ & $\boldsymbol{d}=\mathbf{0}$ & $d=0.2$ & $d=0.4$ & $d=0.6$ & $d=0.8$ & $d=1$ & $d=1.2$ \\
\hline \multicolumn{9}{|l|}{ ARFIMA(0,d,0) } \\
\hline$I S E \tilde{d}_{N}^{(M I R}$ & 0.0911 & .0968 & 0.0988 & 0.0949 & 0.1018 & 0.1022 & 0.0973 & 0.1055 \\
\hline$\sqrt{M S E} \widehat{d}_{N}^{(I R)}$ & 0.1900 & 0.2156 & 0.2229 & 0.2081 & 0.2008 & 0.1806 & 0.1622 & 0.1432 \\
\hline$\sqrt{M S E} \widetilde{d}_{N}^{(M S)}$ & 0.1405 & 0.1441 & 0.1432 & 0.1523 & 0.1681 & 0.1765 & 0.1703 & 0.1643 \\
\hline$\sqrt{M S E} \widehat{d}_{N}^{(A D G)}$ & 0.0764 & 0.0803 & 0.0787 & 0.0838 & 0.0778 & 0.0785 & 0.0800 & 0.0758 \\
\hline$\sqrt{M S E} \widetilde{d}_{N}^{(W A V)}$ & .0716 & 0.0795 & 0.0849 & 0.0865 & 0.0808 & 0.0848 & 0.0701 & 0.0707 \\
\hline \multicolumn{9}{|l|}{$\operatorname{ARFIMA}(1, \mathrm{~d}, 1)$} \\
\hline$\sqrt{M S E} \widetilde{d}_{N}^{(M / R)}$ & 0.1527 & 0.1363 & 0.1315 & 0.1173 & 0.1212 & 0.1099 & 0.1129 & 0.1098 \\
\hline$\sqrt{M S E} \widehat{d}_{N}^{(I R)}$ & 0.2255 & 0.2328 & 0.2217 & 0.2205 & 0.2080 & 0.1773 & 0.1592 & 0.1353 \\
\hline$\sqrt{M S E} \widetilde{d}_{N}^{(M S)}$ & 0.1393 & 0.1448 & 0.1446 & 0.1521 & 0.1668 & 0.1744 & 0.1688 & 0.1625 \\
\hline$\sqrt{M S E} \widehat{d}_{N}^{(A D G)}$ & 0.0939 & 0.0914 & 0.0925 & 0.1012 & 0.0933 & 0.0887 & 0.0897 & 0.0872 \\
\hline$\sqrt{M S E} \widetilde{d}_{N}^{(W A V)}$ & 0.1728 & 0.1625 & 0.1591 & 0.1424 & 0.1373 & 0.1210 & 0.1026 & 0.0922 \\
\hline \multicolumn{9}{|l|}{$X^{(d, 1,0.1)}$} \\
\hline$\sqrt{M S E} \widetilde{d}_{N}^{(M I R)}$ & 0.0892 & 0.1003 & 0.1010 & 0.1093 & 0.1168 & 0.1126 & 0.1158 & 0.1271 \\
\hline$\sqrt{M S E} \widehat{d}_{N}^{(I R)}$ & 0.1803 & 0.2081 & 0.2186 & 0.2062 & 0.2043 & 0.1840 & 0.1700 & 0.1569 \\
\hline$\sqrt{M S E} \widetilde{d}_{N}^{(M S)}$ & 0.1418 & 0.1438 & 0.1425 & 0.1472 & 0.1538 & 0.1680 & 0.1677 & 0.1697 \\
\hline$\sqrt{M S E} \widehat{d}_{N}^{(A D G)}$ & 0.0808 & 0.0836 & 0.0804 & 0.0864 & 0.0817 & 0.0812 & 0.0842 & 0.0817 \\
\hline$\sqrt{M S E} \widetilde{d}_{N}^{(W A V)}$ & 0.0954 & 0.0871 & 0.0891 & 0.0856 & 0.0772 & 0.0757 & 0.0798 & 0.0856 \\
\hline \multicolumn{9}{|l|}{$x^{(d, 0,1)}$} \\
\hline$\sqrt{M S E} \widetilde{d}_{N}^{(M / R)}$ & 0.0915 & 0.0950 & 0.0962 & 0.1018 & 0.1043 & 0.1111 & 0.1104 & 0.1205 \\
\hline$\sqrt{M S E} \widehat{d}_{N}^{(I R)}$ & 0.1839 & 0.2141 & 0.2094 & 0.2179 & 0.2010 & 0.1827 & 0.1625 & 0.1411 \\
\hline$\sqrt{M S E} \widetilde{d}_{N}^{(M S)}$ & 0.1393 & 0.1437 & 0.1446 & 0.1447 & 0.1524 & 0.1709 & 0.1721 & 0.1708 \\
\hline$\sqrt{M S E} \widehat{d}_{N}^{(A D G)}$ & 0.0746 & 0.0790 & 0.0750 & 0.0808 & 0.0778 & 0.0779 & 0.0754 & 0.0780 \\
\hline$\sqrt{M S E} \widetilde{d}_{N}^{(W A V)}$ & 0.0756 & 0.0786 & 0.0767 & 0.0750 & 0.0667 & 0.0724 & 0.0789 & 0.0846 \\
\hline \multicolumn{9}{|l|}{$X^{(d, \log )}$} \\
\hline$\sqrt{M S E} \widetilde{d}_{N}^{(M}$ & 0.0836 & 0.1064 & 0.1089 & 0.1161 & 0.1138 & 0.1197 & 0.1252 & 0.1380 \\
\hline$\sqrt{M S E} \widetilde{d}_{N}^{(I R)}$ & 0.1810 & 0.2100 & 0.2089 & 0.2009 & 0.1853 & 0.1819 & 0.1666 & 0.1542 \\
\hline$\sqrt{M S E} \widetilde{d}_{N}^{(M S)}$ & 0.1500 & 0.1529 & 0.1564 & 0.1677 & 0.1649 & 0.1654 & 0.1660 & 0.1578 \\
\hline$\sqrt{M S E} \widehat{d}_{N}^{(A D G)}$ & 0.0822 & 0.0864 & 0.0844 & 0.0901 & 0.0827 & 0.0797 & 0.0852 & 0.0846 \\
\hline $\begin{array}{l}\sqrt{M S E} \widetilde{d}_{N}^{(W A V)} \\
X^{(\text {trend })}\end{array}$ & 0.0974 & 0.1087 & 0.0996 & 0.1068 & 0.1007 & 0.1031 & 0.0967 & 0.0829 \\
\hline$\sqrt{M S E} \widetilde{d}_{N}^{(M I I}$ & 0.4684 & 0.2922 & 0.1633 & 0.1051 & 27 & 0.1176 & 0.1176 & 0.1279 \\
\hline$\sqrt{M S E} \widetilde{d}_{N}^{(I R)}$ & 0.2793 & 0.2192 & 0.2048 & 0.2029 & 0.1964 & 0.1824 & 0.1616 & 0.1443 \\
\hline$\sqrt{M S E} \widetilde{d}_{N}^{(M S)}$ & 0.9077 & 0.6067 & 0.3444 & 0.2150 & 0.2024 & 0.1994 & 0.1683 & 0.1471 \\
\hline$\sqrt{M S E} \widehat{d}_{N}^{(A D G)}$ & 0.5674 & 0.3564 & 0.1787 & 0.1009 & 0.0845 & 0.0901 & 0.0880 & 0.0878 \\
\hline$\sqrt{M S E} \widetilde{d}_{N}^{(W /}$ & 0.0961 & 0.0908 & 0.0886 & 0.0913 & 0.0917 & 0.0907 & 0.0804 & 0.0896 \\
\hline
\end{tabular}


Table 3: Comparison between $\widetilde{d}_{N}^{(M / R)}$ and other famous semiparametric estimators of $d\left(\widetilde{d}_{N}^{(I R)}\right.$, $\widetilde{d}_{N}^{(M S)}, \widehat{d}_{N}^{(A D G)}$ and $\widetilde{d}_{N}^{(W A V)}$ ) applied to fractionally integrated processes for $N=5000$, several values of $d \in(-0.5,1.25)$ and 1000 independent replications.

\begin{tabular}{|c|c|c|c|c|c|c|c|c|}
\hline$N=5000$ & $=-0.2$ & $d=0$ & $d=0.2$ & $I=0.4$ & $d=.6$ & $d=0.8$ & $d=1$ & $d=1.2$ \\
\hline \multicolumn{9}{|l|}{$\operatorname{ARFIMA}(0, \mathrm{~d}, 0)$} \\
\hline$\overline{S E} \tilde{d}_{N}^{(M I}$ & 0.0391 & 0.0318 & 0.0329 & 0.0346 & 0.0363 & 0.0381 & 0.0399 & 0.0513 \\
\hline$\sqrt{M S E} \widetilde{d}_{N}^{(I R)}$ & 0.0652 & 0.0637 & 0.0636 & 0.0636 & 0.0591 & 0.0574 & 0.0499 & 0.0477 \\
\hline$\sqrt{M S E} \widetilde{d}_{N}^{(M S)}$ & 0.0428 & 0.0434 & 0.0425 & 0.0447 & 0.0483 & 0.0587 & 0.0447 & 0.1419 \\
\hline$\sqrt{M S E} \widehat{d}_{N}^{(A D G)}$ & 0.0326 & 0.0323 & 0.0324 & 0.0341 & 0.0341 & 0.0334 & 0.0333 & 0.0327 \\
\hline$\sqrt{M S E} \widetilde{d}_{N}^{(W A V)}$ & 0.0313 & 0.0305 & 0.0269 & 0.0308 & 0.0329 & 0.0356 & 0.0340 & 0.0350 \\
\hline \multicolumn{9}{|l|}{ ARFIMA(1,d,1) } \\
\hline$\sqrt{M S E} \widetilde{d}_{N}^{(M I}$ & 0.0756 & 0.0666 & 0.0605 & 0.0551 & 0.0518 & 0.0514 & 0.0557 & 0.0585 \\
\hline$\sqrt{M S E} \widetilde{d}_{N}^{(I R)}$ & 0.1141 & 0.0901 & 0.0792 & 0.0730 & 0.0612 & 0.0559 & 0.0491 & 0.0423 \\
\hline$\sqrt{M S E} \widetilde{d}_{N}^{(M S)}$ & 0.0425 & 0.0437 & 0.0428 & 0.0449 & 0.0481 & 0.0584 & 0.0444 & 0.1417 \\
\hline$\sqrt{M S E} \widehat{d}_{N}^{(A D G)}$ & 0.0333 & 0.0335 & 0.0336 & 0.0364 & 0.0359 & 0.0346 & 0.0338 & 0.0340 \\
\hline$\sqrt{M S E} \widetilde{d}_{N}^{(W A V)}$ & 0.0566 & 0.0603 & 0.0545 & 0.0560 & 0.0546 & 0.0545 & 0.0493 & 0.0474 \\
\hline \multicolumn{9}{|l|}{$X^{(d, 1,0.1)}$} \\
\hline$\sqrt{M S E} \widetilde{d}_{N}^{(M / R)}$ & 0.0302 & 0.0401 & 0.0412 & 0.0465 & 0.0427 & 0.0444 & 0.0456 & 0.0490 \\
\hline$\sqrt{M S E} \widetilde{d}_{N}^{(I R)}$ & 0.0606 & 0.0678 & 0.0773 & 0.0740 & 0.0652 & 0.0568 & 0.0554 & 0.0472 \\
\hline$\sqrt{M S E} \widetilde{d}_{N}^{(M S)}$ & 0.0429 & 0.0483 & 0.0486 & 0.0502 & 0.0447 & 0.0523 & 0.0458 & 0.1322 \\
\hline$\sqrt{M S E} \widehat{d}_{N}^{(A D G)}$ & 0.0390 & 0.0410 & 0.0400 & 0.0395 & 0.0357 & 0.0378 & 0.0404 & 0.0363 \\
\hline$\sqrt{M S E} \widetilde{d}_{N}^{(W A V)}$ & 0.0363 & 0.0393 & 0.0406 & 0.0375 & 0.0340 & 0.0408 & 0.0406 & 0.0444 \\
\hline \multicolumn{9}{|l|}{$X^{(d, 0,1)}$} \\
\hline$\sqrt{M S E} \widetilde{d}_{N}^{(M}$ & 0.0330 & 0.0297 & 0.0314 & 0.0320 & 0.0319 & 0.0315 & 0.0339 & 0.0395 \\
\hline$\sqrt{M S E} \widetilde{d}_{N}^{(I R)}$ & 0.0642 & 0.0652 & 0.0693 & 0.0633 & 0.0630 & 0.0560 & 0.0478 & 0.0428 \\
\hline$\sqrt{M S E} \widetilde{d}_{N}^{(M S)}$ & 0.0432 & 0.0422 & 0.0461 & 0.0434 & 0.0489 & 0.0547 & 0.0437 & 0.1263 \\
\hline$\sqrt{M S E} \widehat{d}_{N}^{(A D G)}$ & 0.0318 & 0.0318 & 0.0322 & 0.0345 & 0.0361 & 0.0321 & 0.0327 & 0.0326 \\
\hline$\sqrt{M S E} \widetilde{d}_{N}^{(W A V)}$ & 0.0271 & 0.0298 & 0.0248 & 0.0319 & 0.0348 & 0.0331 & 0.0384 & 0.0402 \\
\hline \multicolumn{9}{|l|}{$x^{(d, \log )}$} \\
\hline$\sqrt{M S E} \widetilde{d}_{N}^{(M I R)}$ & 0.0370 & 0.0345 & 0.0383 & 0.0461 & 0.0461 & 0.0519 & 0.0555 & 0.0608 \\
\hline$\sqrt{M S E} \widetilde{d}_{N}^{(I R)}$ & 0.0627 & 0.0683 & 0.0676 & 0.0659 & 0.0587 & 0.0528 & 0.0490 & 0.0501 \\
\hline$\sqrt{M S E} \widetilde{d}_{N}^{(M S)}$ & 0.0582 & 0.0615 & 0.0632 & 0.0640 & 0.0636 & 0.0584 & 0.0552 & 0.1300 \\
\hline$\sqrt{M S E} \widehat{d}_{N}^{(A D G)}$ & 0.0417 & 0.0427 & 0.0414 & 0.0411 & 0.0401 & 0.0405 & 0.0415 & 0.0403 \\
\hline$\sqrt{M S E} \widetilde{d}_{N}^{(W A V)}$ & 0.0604 & 0.0618 & 0.0589 & 0.0609 & 0.0632 & 0.0600 & 0.0609 & 0.0669 \\
\hline \multicolumn{9}{|l|}{$X^{(\text {trend })}$} \\
\hline$\sqrt{M S E} \widetilde{d}_{N}^{(M / R)}$ & 0.0720 & 0.0372 & 0.0349 & 0.0363 & 0.0363 & 0.0380 & 0.0450 & 0.0864 \\
\hline$\sqrt{M S E} \tilde{d}_{N}^{(I R)}$ & 0.0677 & 0.0639 & 0.0690 & 0.0655 & 0.0602 & 0.0545 & 0.0485 & 0.0506 \\
\hline$\sqrt{M S E} \widetilde{d}_{N}^{(M S)}$ & 0.7760 & 0.6067 & 0.1480 & 0.0675 & 0.0680 & 0.0750 & 0.0443 & 0.1512 \\
\hline$\sqrt{M S E} \widehat{d}_{N}^{(A D G)}$ & 0.6019 & 0.3613 & 0.1502 & 0.0555 & 0.0387 & 0.0377 & 0.0369 & 0.0364 \\
\hline$\sqrt{M S E} \tilde{d}_{N}^{(W A V)}$ & 0.4988 & 0.0623 & 0.0389 & 0.0344 & 0.0362 & 0.0402 & 0.0422 & 0.0444 \\
\hline
\end{tabular}


Table 4: Comparison between $\widetilde{d}_{N}^{(M I R)}$ and other famous semiparametric estimators of $d\left(\widetilde{d}_{N}^{(I R)}\right.$, $\widetilde{d}_{N}^{(M S)}, \widehat{d}_{N}^{(A D G)}$ and $\widetilde{d}_{N}^{(\text {WAV })}$ )applied to fractionally integrated processes for $N=50000$, several values of $d \in(-0.5,1.25)$ and 1000 independent replications.

\begin{tabular}{|c|c|c|c|c|c|c|c|c|}
\hline$N=50000$ & $=-0.2$ & $d=0$ & $=0.2$ & $d=0.4$ & $d=0.6$ & $d=0.8$ & $d=1$ & $d=1.2$ \\
\hline \multicolumn{9}{|l|}{$\operatorname{ARFIMA}(0, d, 0)$} \\
\hline$\overline{S E} \widetilde{d}_{N}^{(M I R}$ & 0.0201 & 0.0081 & 0.0132 & 0.0141 & 0.0139 & 0.0150 & 0.0128 & 0.0294 \\
\hline$\sqrt{M S E} \widetilde{d}_{N}^{(I R)}$ & 0.0248 & 0.0219 & 0.0218 & 0.0216 & 0.0191 & 0.0203 & 0.0138 & 0.0144 \\
\hline$\sqrt{M S E} \widetilde{d}_{N}^{(M S)}$ & 0.0151 & 0.0161 & 0.0150 & 0.0127 & 0.0178 & 0.0217 & 0.0127 & 0.1595 \\
\hline$\sqrt{M S E} \widehat{d}_{N}^{(A D G)}$ & 0.0143 & 0.0150 & 0.0160 & 0.0134 & 0.0159 & 0.0133 & 0.0139 & 0.0149 \\
\hline$\sqrt{M S E} \widetilde{d}_{N}^{(W A V)}$ & 0.0102 & 0.0079 & 0.0086 & 0.0102 & 0.0107 & 0.0196 & 0.0183 & 0.0253 \\
\hline \multicolumn{9}{|l|}{$\operatorname{ARFIMA}(1, d, 1)$} \\
\hline$\sqrt{M S E} \widetilde{d}_{N}^{(M I}$ & 0.0440 & 0.0278 & 0.0247 & 0.0232 & 0.0185 & 0.0233 & 0.0198 & 0.0326 \\
\hline$\sqrt{M S E} \widetilde{d}_{N}^{(I R)}$ & 0.0906 & 0.0658 & 0.0479 & 0.0355 & 0.0298 & 0.0230 & 0.0194 & 0.0163 \\
\hline$\sqrt{M S E} \widetilde{d}_{N}^{(M S)}$ & 0.0146 & 0.0125 & 0.0142 & 0.0163 & 0.0179 & 0.0257 & 0.0141 & 0.1564 \\
\hline$\sqrt{M S E} \widehat{d}_{N}^{(A D G)}$ & 0.0160 & 0.0137 & 0.0144 & 0.0160 & 0.0156 & 0.0158 & 0.0154 & 0.0138 \\
\hline$\sqrt{M S E} \widetilde{d}_{N}^{(W A V)}$ & 0.0233 & 0.0252 & 0.0268 & 0.0210 & 0.0179 & 0.0257 & 0.0254 & 0.0319 \\
\hline \multicolumn{9}{|l|}{$X^{(d, 1,0.1)}$} \\
\hline$\sqrt{M S E} \widetilde{d}_{N}^{(M / R)}$ & 0.0093 & 0.0243 & 0.0268 & 0.0273 & 0.0280 & 0.0265 & 0.0249 & 0.0224 \\
\hline$\sqrt{M S E} \widetilde{d}_{N}^{(I R)}$ & 0.0182 & 0.0330 & 0.0349 & 0.0342 & 0.0335 & 0.0316 & 0.0258 & 0.0267 \\
\hline$\sqrt{M S E} \widetilde{d}_{N}^{(M S)}$ & 0.0244 & 0.0293 & 0.0267 & 0.0276 & 0.0251 & 0.0216 & 0.0216 & 0.1375 \\
\hline$\sqrt{M S E} \widehat{d}_{N}^{(A D G)}$ & 0.0243 & 0.0283 & 0.0257 & 0.0265 & 0.0230 & 0.0248 & 0.0244 & 0.0253 \\
\hline$\sqrt{M S E} \widetilde{d}_{N}^{(W A V)}$ & 0.0232 & 0.0290 & 0.0273 & 0.0397 & 0.0290 & 0.0281 & 0.0228 & 0.0318 \\
\hline \multicolumn{9}{|l|}{$x^{(d, o, 1)}$} \\
\hline$\sqrt{M S E} \widetilde{d}_{N}^{(M I R)}$ & 0.0181 & 0.089 & 0.0107 & 0.0110 & 0.0108 & 0.0125 & 0.0115 & 0.0121 \\
\hline$\sqrt{M S E} \widetilde{d}_{N}^{(I R)}$ & 0.0273 & 0.0205 & 0.0236 & 0.0215 & 0.0221 & 0.0159 & 0.0147 & 0.0131 \\
\hline$\sqrt{M S E} \widetilde{d}_{N}^{(M S)}$ & 0.0140 & 0.0154 & 0.0151 & 0.0166 & 0.0167 & 0.0227 & 0.0159 & 0.1337 \\
\hline$\sqrt{M S E} \widehat{\sim}_{N}^{(A D G)}$ & 0.0148 & 0.0165 & 0.0167 & 0.0177 & 0.0146 & 0.0145 & 0.0161 & 0.0154 \\
\hline$\sqrt{M S E} \widetilde{d}_{N}^{(W A V)}$ & 0.0099 & 0.0167 & 0.0135 & 0.0156 & 0.0189 & 0.0148 & 0.0283 & 0.0268 \\
\hline \multicolumn{9}{|l|}{$X^{(d, \log )}$} \\
\hline$\sqrt{M S E} \widetilde{d}_{N}^{(M / R)}$ & 0.0193 & 0.0240 & 0.0287 & 0.0312 & 0.0382 & 0.0390 & 0.0419 & 0.0472 \\
\hline$\sqrt{M S E} \widetilde{d}_{N}^{(I R)}$ & 0.0300 & 0.0256 & 0.0282 & 0.0294 & 0.0210 & 0.0191 & 0.0244 & 0.0305 \\
\hline$\sqrt{M S E} \widetilde{d}_{N}^{(M S)}$ & 0.0463 & 0.0498 & 0.0480 & 0.0504 & 0.0478 & 0.0408 & 0.0418 & 0.1480 \\
\hline$\sqrt{M S E} \widehat{d}_{N}^{(A D G)}$ & 0.0456 & 0.0475 & 0.0464 & 0.0464 & 0.0438 & 0.0456 & 0.0468 & 0.0453 \\
\hline$\sqrt{M S E} \widetilde{d}_{N}^{(W A V)}$ & 0.0529 & 0.0515 & 0.0509 & 0.0524 & 0.0465 & 0.0468 & 0.0544 & 0.0498 \\
\hline \multicolumn{9}{|l|}{$X^{(\text {trend })}$} \\
\hline$\sqrt{M S E} \widetilde{d}_{N}^{(M / R)}$ & 0.0271 & 0.0097 & 0.0127 & 0.0130 & 0.0132 & 0.0132 & 0.0126 & 0.0562 \\
\hline$\sqrt{M S E} \widetilde{d}_{N}^{(I R)}$ & 0.0282 & 0.0228 & 0.0226 & 0.0211 & 0.0199 & 0.0160 & 0.0165 & 0.0194 \\
\hline$\sqrt{M S E} \widetilde{d}_{N}^{(M S)}$ & 0.9840 & 0.6253 & 0.1134 & 0.0194 & 0.0224 & 0.0395 & 0.0117 & 0.1655 \\
\hline$\sqrt{M S E} \widehat{d}_{N}^{(A D G)}$ & 0.6190 & 0.3616 & 0.1356 & 0.0209 & 0.0158 & 0.0156 & 0.0153 & 0.0155 \\
\hline$\sqrt{M S E} \widetilde{d}_{N}^{(W A V)}$ & 1.0023 & 0.5575 & 0.0386 & 0.0182 & 0.0181 & 0.0253 & 0.0474 & 0.0275 \\
\hline
\end{tabular}


(KPSS and V/S) or non-stationarity (A DF and PP) tests (see more details on these tests in the previous section).

As it is suggested for the corresponding R-software commands (see also Banerjee et al. 1993), we chose the following trimming parameters for the classical tests:

- $\quad K=\left[\frac{3}{13} \sqrt{2}\right]$ for KPSS TEST;

- $K=\sqrt{N}$ for V/S test;

- $K=\left[\sqrt{(N-1)^{1 / 3}}\right]$ for PP test;

- $K=\left[4\left(\frac{N}{100}\right)^{1 / 4}\right]$ for PP test;

The results of these simulations with a type I error classically chosen to 0.05 are provided in Tables 5, 6, 7 and 8.

Conclusions of simulations: As it is well known, from their constructions, KPSS, V/S, ADF and PP tests should asymptotically decide the stationarity hypothesis when $d=0$, and the non-stationarity hypothesis when $d>0$. It was exactly what we observe in these simulations. For ARIMA $(p, d, 0)$ processes with $d=0$ or $d=1$ (i. e. AR(1) process when $d=0$ ), $\mathrm{ADF}$ and PP tests are more efficient tests than our adaptive MIR tests when $N=500$. However, note that all stationarity tests do not control the size for $\phi=-0.9$. But when $N=5000$ the tests computed from $\widetilde{d}_{N}^{(M I R)}$ provide comparable and convincing results. Note also that KPSS and V/S provide reasonable results but less efficient than the other tests. In case of processes with $d \in(0,1)$, the tests computed from $\widetilde{d}_{N}^{(M I R)}$ obtain clearly better performances than classical non-stationarity tests ADF or PP which accept the non-stationarity assumption $H_{0}^{\prime}$ even if the processes are stationary when $0<d$ $<0.5$ for instance. The case of the V/S test is different since this test is built for distinguishing between short and long-memory processes. However, as it was already established in Giraitis et al. (2003), V/S test is slightly more accurate than KPSS for testing the stationarity. Note also that a renormalized version of this test has been defined in Giraitis et al. (2006) for taking into account the value of $d$.

\subsubsection{Comparison of MIR Fractional Unit Roots test $\widetilde{F}_{N}$ and Dolado et al. and Lobato and Velasco Fractional Unit Roots tests}

Monte-Carlo experiments were also done for evaluating the performances of new Fractional Unit Root test $\tilde{F}_{N}$ and for comparing it to the Fractional Unit Roots tests defined in Dolado et al. (2002) and in Lobato and Velasco (2007).

1. The student-type test statistic defined in Dolado et al. (2002) is such as: 


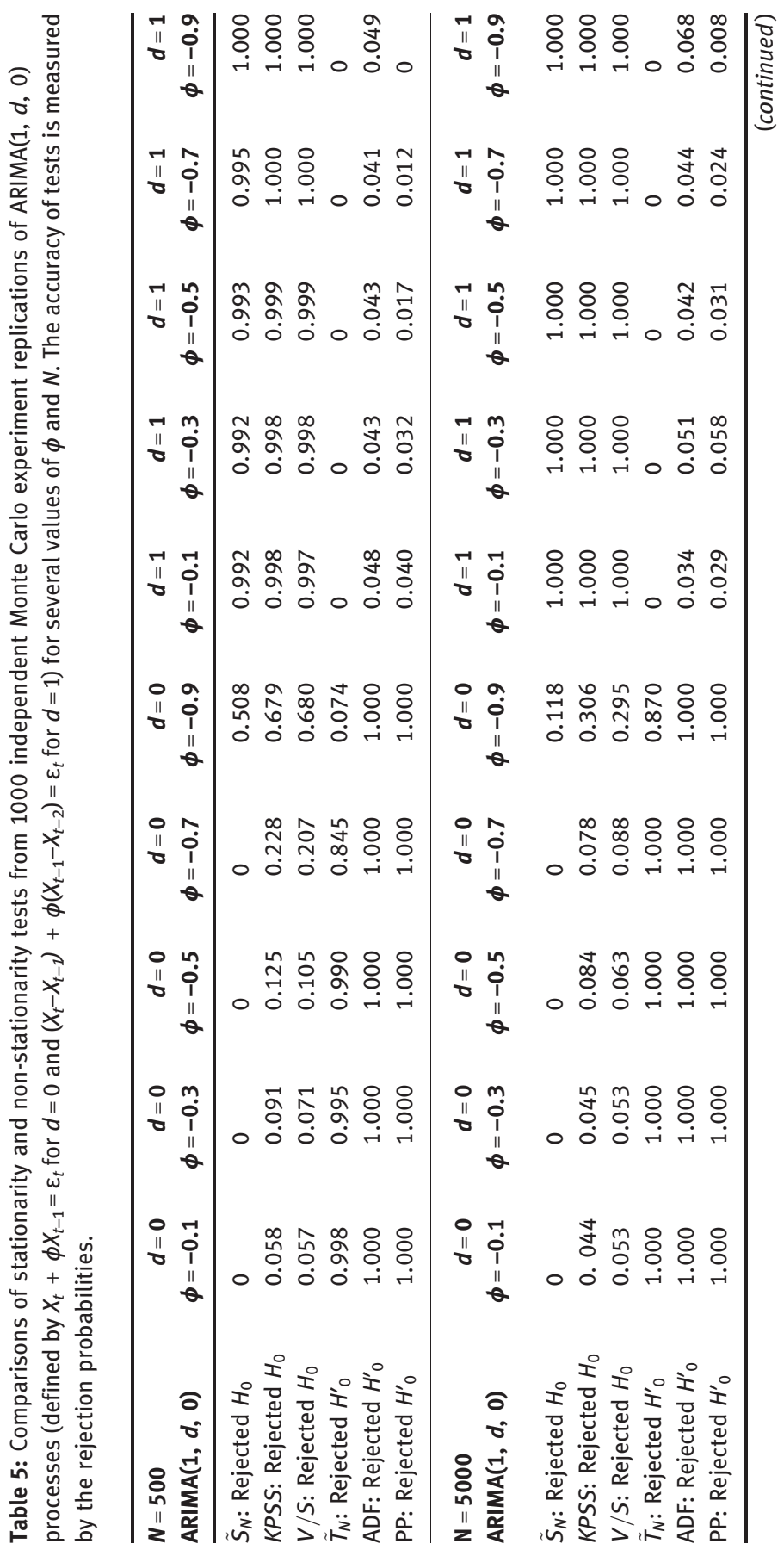




\begin{tabular}{|c|c|c|c|c|}
\hline $\begin{array}{cc}-1 & 0 \\
& 0 \\
0 & 1 \\
& \\
0 & 0\end{array}$ & 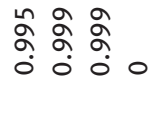 & 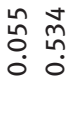 & $\begin{array}{ll}1 & 9 \\
11 & 0 \\
0 & 1 \\
& \| \\
0 & 0\end{array}$ & 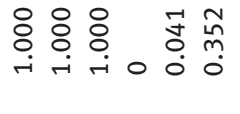 \\
\hline 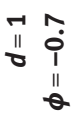 & 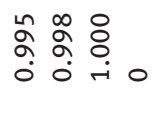 & 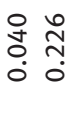 & $\begin{array}{ll}\pi & \hat{0} \\
0 & 1 \\
0 & 11 \\
& 0\end{array}$ & 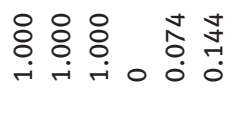 \\
\hline $\begin{array}{cc}\pi & n \\
11 & 0 \\
0 & 1 \\
& \| \\
0 & 0\end{array}$ & 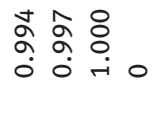 & $\begin{array}{ll}\infty & \infty \\
m & 0 \\
0 & \stackrel{?}{!} \\
0 & 0\end{array}$ & 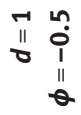 & 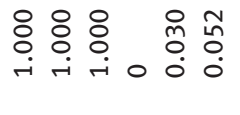 \\
\hline 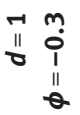 & 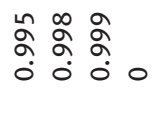 & \begin{tabular}{ll}
$\infty$ & $N$ \\
\multirow{0}{*}{} & $N$ \\
0 & 0 \\
0
\end{tabular} & $\begin{array}{ll}71 & m \\
0 & 0 \\
0 & 1 \\
& 11 \\
\theta & 0\end{array}$ & 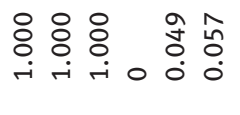 \\
\hline $\begin{array}{cc}\pi & -1 \\
0 & 0 \\
0 & 1 \\
0 & 0\end{array}$ & 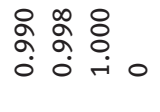 & $\begin{array}{ll}0 & -1 \\
0 & 0 \\
0 & 0 \\
0 & 0\end{array}$ & $\begin{array}{ll}7 & -1 \\
& 0 \\
0 & 1 \\
& 11 \\
& 0\end{array}$ & 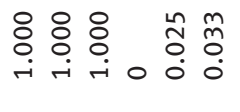 \\
\hline $\begin{array}{cc}0 & 9 \\
0 & 0 \\
0 & 1 \\
11 \\
0\end{array}$ & 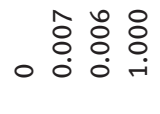 & \begin{tabular}{ll}
8 & 8 \\
0 & 0 \\
\hdashline & 0 \\
$-i$
\end{tabular} & $\begin{array}{ll}0 & a \\
0 & 0 \\
0 & 1 \\
& 11 \\
& 0\end{array}$ & 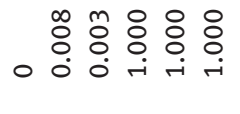 \\
\hline $\begin{array}{cc}0 & \\
0 & 0 \\
0 & 1 \\
& 11 \\
0 & 0\end{array}$ & 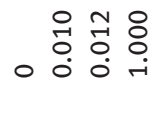 & \begin{tabular}{ll}
8 & 8 \\
\hdashline & 0 \\
$-i$ & - \\
$-i$
\end{tabular} & $\begin{array}{ll}0 & 1 \\
0 & 0 \\
0 & 1 \\
& 11 \\
0 & 0\end{array}$ & 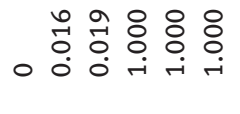 \\
\hline $\begin{array}{cc}0 & n \\
0 & 0 \\
0 & 1 \\
11 \\
0\end{array}$ & 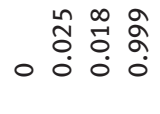 & \begin{tabular}{ll}
8 & 8 \\
0 & 0 \\
\hdashline & 0 \\
$-i$
\end{tabular} & $\begin{array}{cc}0 & n \\
0 & 0 \\
0 & 1 \\
& 11 \\
& \\
& 0\end{array}$ & 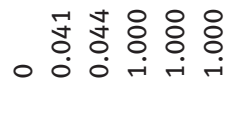 \\
\hline $\begin{array}{cc}0 & m \\
0 & 0 \\
0 & i \\
& 11 \\
0 & 0\end{array}$ & $\begin{array}{l}\text { సे } \\
\text { ô } \\
0\end{array}$ & \begin{tabular}{ll}
$\circ$ & $\circ$ \\
\hdashline
\end{tabular} & $\begin{array}{cc}0 & m \\
0 & 0 \\
0 & 1 \\
& 11 \\
& 0\end{array}$ & 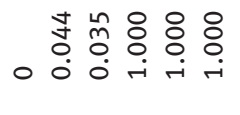 \\
\hline $\begin{array}{cc}0 & -1 \\
0 & 0 \\
0 & 1 \\
11 \\
0 \\
0\end{array}$ & 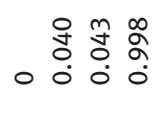 & \begin{tabular}{ll}
8 & 8 \\
\hdashline & 0 \\
& 0 \\
$-i$
\end{tabular} & $\begin{array}{cc}0 & -1 \\
0 & 0 \\
0 & 1 \\
& 11 \\
& \\
& 0\end{array}$ & 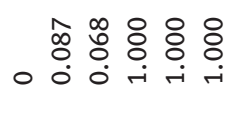 \\
\hline 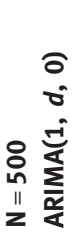 & 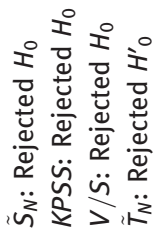 & 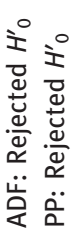 & 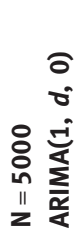 & 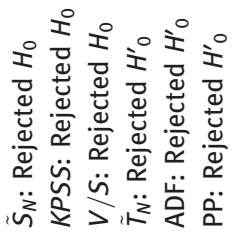 \\
\hline
\end{tabular}


Table 6: Comparisons of stationarity and non-stationarity tests from 1000 independent Monte Carlo experiment replications of $\operatorname{ARFIMA}(0, d, 0)$ processes for several values of $d$ and $N$. The accuracy of tests is measured by the rejection probabilities.

\begin{tabular}{|c|c|c|c|c|c|c|c|c|}
\hline $\begin{array}{l}\mathrm{N}=500 \\
\operatorname{ARFIMA}(0, d, 0)\end{array}$ & $d=-0.2$ & $\boldsymbol{d}=\mathbf{0}$ & $d=0.2$ & $d=0.4$ & $d=0.6$ & $d=0.8$ & $d=1$ & $d=1.2$ \\
\hline$\tilde{S}_{N}:$ Rejected $H_{0}$ & 0 & 0 & 0 & 0.003 & 0.276 & 0.917 & 0.998 & 0.999 \\
\hline KPSS: Rejected $H_{0}$ & 0 & 0.059 & 0.395 & 0.771 & 0.946 & 0.989 & 0.999 & 0.999 \\
\hline$V / S$ : Rejected $H_{0}$ & 0 & 0.052 & 0.446 & 0.847 & 0.970 & 0.993 & 0.998 & 1.000 \\
\hline$\tilde{T}_{N}:$ Rejected $H_{0}^{\prime}$ & 1.000 & 1.000 & 0.965 & 0.421 & 0.017 & 0 & 0 & 0 \\
\hline ADF: Rejected $H_{0}^{\prime}$ & 1.000 & 1.000 & 1.000 & 0.977 & 0.615 & 0.233 & 0.065 & 0.005 \\
\hline PP: Rejected $H_{0}^{\prime}$ & 1.000 & 1.000 & 1.000 & 1.000 & 0.919 & 0.447 & 0.065 & 0.002 \\
\hline $\begin{array}{l}\mathrm{N}=5000 \\
\operatorname{ARFIMA}(0, d, 0)\end{array}$ & $d=-0.2$ & $\boldsymbol{d}=\mathbf{0}$ & $d=0.2$ & $d=0.4$ & $d=0.6$ & $d=0.8$ & $d=1$ & $d=1.2$ \\
\hline$\tilde{S}_{N}:$ Rejected $H_{0}$ & 0 & 0 & 0 & 0 & 0.912 & 1.000 & 1.000 & 1.000 \\
\hline KPSS: Rejected $H_{0}$ & 0 & 0.042 & 0.674 & 0.996 & 1.000 & 1.000 & 1.000 & 1.000 \\
\hline$\underset{\sim}{V / S}$ : Rejected $H_{0}$ & 0 & 0.038 & 0.694 & 0.992 & 1.000 & 1.000 & 1.000 & 1.000 \\
\hline$\tilde{T}_{N}:$ Rejected $H_{0}^{\prime}$ & 1.000 & 1.000 & 1.000 & 0.946 & 0 & 0 & 0 & 0 \\
\hline ADF: Rejected $H_{0}^{\prime}$ & 1.000 & 1.000 & 1.000 & 1.000 & 0.946 & 0.448 & 0.050 & 0.004 \\
\hline PP: Rejected $H^{\prime}{ }_{0}$ & 1.000 & 1.000 & 1.000 & 1.000 & 1.000 & 0.705 & 0.042 & 0 \\
\hline
\end{tabular}

$$
\widehat{T}_{D G M}=\frac{\sum_{t=2}^{N}\left(x_{t}-x_{t-1}\right) \Delta^{\hat{d}_{1}} X_{t-1}}{\left(\sum_{t=2}^{N}\left(\Delta^{\widehat{d}_{1}} X_{t-1}\right)^{2} \times \frac{1}{N} \sum_{t=2}^{N}\left(X_{t}-X_{t-1}-\widehat{\phi} \Delta^{{ }^{1}} X_{t-1}\right)^{2}\right)^{1 / 2}},
$$

with $\widehat{\phi}=\frac{\sum_{t=2}^{N}\left(X_{t}-X_{t-1}\right) \Delta^{\hat{d}_{1}} X_{t-1}}{\sum_{t=2}^{N}\left(\Delta^{\hat{d}_{1}} X_{t-1}\right)^{2}}$ and $\widehat{d}_{1}$ is the minimum between an ordinary least square stimator of $d_{1}$ and 1-c with $c>0$ small enough (typically $c=0.02$ ). This is an extension in a fractional framework of the Dickey-Fuller test.

2. The efficient Wald test statistic defined in Lobato and Velasco (2007) is based on a two-step student test of a regression coefficient, i. e. $\widehat{T}_{L V}$ is the student test of the $\left(z_{t}\right)_{t}$ coefficient for the regression of $X_{t}-X_{t-1}$ onto variables $z_{t}-\widehat{\alpha}_{1} z_{t-1}-\ldots$ $-\widehat{\alpha}_{p} z_{t-p}, X_{t-1}-X_{t-2}, \ldots, X_{t-p}-X_{t-p-1}$ for $t=p+1, \ldots, n$, where $z_{t}$ is defined as

$$
z_{t}=\frac{\Delta^{\hat{d}} X_{t}-\left(X_{t}-X_{t-1}\right)}{1-\hat{d}}
$$

and where $\left(\hat{\alpha}_{1}, \ldots, \hat{\alpha}_{p}\right)$ are obtained as a minimizer of $\sum_{k=p}^{n}\left(\Delta^{\hat{d}} X_{t}-\right.$ $\left.\alpha_{1} \Delta^{\hat{d}} X_{t-1}-\ldots-\alpha_{p} \Delta^{\hat{d}} X_{t-p}\right)^{2}$ and $\widehat{d}$ is a semi-parametric local Whittle type estimator of $d$. Note that $\widehat{T}_{L V}$ is depending on $p$ and in the sequel we will chose $p=1$ and $p=10$, defining respectively $\widehat{T}_{L V 1}$ and $\widehat{T}_{L V 10}$. 
Table 7: Comparisons of stationarity and non-stationarity tests from 1000 independent Monte Carlo experiment replications of $\operatorname{ARFIMA}(1, d, 1)$ processes with $\phi=-0.3$ and $\theta=0.7$ for several values of $d$ and $N$. The accuracy of tests is measured by the rejection probabilities.

\begin{tabular}{|c|c|c|c|c|c|c|c|c|}
\hline $\begin{array}{l}\mathrm{N}=500 \\
\operatorname{ARFIMA}(1, d, 1) \\
\phi=-0.3 ; \theta=0.7\end{array}$ & $d=-0.2$ & $\boldsymbol{d}=\mathbf{0}$ & $d=0.2$ & $d=0.4$ & $d=0.6$ & $d=0.8$ & $d=1$ & $d=1.2$ \\
\hline$\tilde{S}_{N}:$ Rejected $H_{0}$ & 0 & 0 & 0 & 0.015 & 0.442 & 0.898 & 0.987 & 0.999 \\
\hline KPSS: Rejected $H_{0}$ & 0 & 0.079 & 0.454 & 0.836 & 0.959 & 0.995 & 0.997 & 0.999 \\
\hline$\underset{\sim}{V} / S:$ Rejected $H_{0}$ & 0.001 & 0.077 & 0.481 & 0.876 & 0.974 & 0.993 & 1.000 & 1.000 \\
\hline$\tilde{T}_{N}^{\prime}:$ Rejected $H_{0}^{\prime}$ & 0.999 & 0.990 & 0.823 & 0.212 & 0.009 & 0 & 0 & 0 \\
\hline ADF: Rejected $H_{0}^{\prime}$ & 1.000 & 1.000 & 1.000 & 0.961 & 0.623 & 0.230 & 0.056 & 0.010 \\
\hline PP: Rejected $H_{0}^{\prime}$ & 1.000 & 1.000 & 1.000 & 0.999 & 0.781 & 0.270 & 0.036 & 0 \\
\hline $\begin{array}{l}\mathrm{N}=5000 \\
\text { ARFIMA(1, } d, 1) \\
\phi=-0.3 ; \theta=0.7\end{array}$ & $d=-0.2$ & $\boldsymbol{d}=\mathbf{0}$ & $d=0.2$ & $d=0.4$ & $d=0.6$ & $d=0.8$ & $d=1$ & $d=1.2$ \\
\hline$\tilde{S}_{N}:$ Rejected $H_{0}$ & 0 & 0 & 0 & 0.004 & 0.846 & 1.000 & 1.000 & 1.000 \\
\hline KPSS: Rejected $H_{0}$ & 0 & 0.060 & 0.689 & 0.963 & 0.996 & 1.000 & 1.000 & 1.000 \\
\hline$\underset{\sim}{V} / S:$ Rejected $H_{0}$ & 0 & 0.060 & 0.697 & 0.989 & 1.000 & 1.000 & 1.000 & 1.000 \\
\hline$\tilde{T}_{N}^{\prime}:$ Rejected $H_{0}^{\prime}$ & 1.000 & 1.000 & 1.000 & 0.700 & 0.008 & 0 & 0 & 0 \\
\hline ADF: Rejected $H_{0}^{\prime}$ & 1.000 & 1.000 & 1.000 & 1.000 & 0.951 & 0.371 & 0.052 & 0.004 \\
\hline PP: Rejected $H_{0}^{\prime}$ & 1.000 & 1.000 & 1.000 & 1.000 & 0.996 & 0.584 & 0.038 & 0 \\
\hline
\end{tabular}

Table 8: Comparisons of stationarity and non-stationarity tests from 1000 independent Monte Carlo experiment replications of $X^{(d, 0,1)}$ process for several values of $d$ and $N$. The accuracy of tests is measured by the rejection probabilities.

\begin{tabular}{|c|c|c|c|c|c|c|c|c|}
\hline $\begin{array}{l}\mathrm{N}=500 \\
X^{(d, 0,1)}\end{array}$ & $d=-0.2$ & $\boldsymbol{d}=\mathbf{0}$ & $d=0.2$ & $d=0.4$ & $d=0.6$ & $d=0.8$ & $d=1$ & $d=1.2$ \\
\hline$\tilde{S}_{N}:$ Rejected $H_{0}$ & 0 & 0 & 0 & 0.001 & 0.294 & 0.883 & 0.990 & 0.999 \\
\hline KPSS: Rejected $H_{0}$ & 0 & 0.052 & 0.433 & 0.801 & 0.939 & 0.988 & 0.999 & 1.000 \\
\hline$V / S:$ Rejected $H_{0}$ & 0 & 0.035 & 0.464 & 0.844 & 0.963 & 0.995 & 0.999 & 1.000 \\
\hline$\tilde{T}_{N}^{\prime}:$ Rejected $H_{0}^{\prime}$ & 1.000 & 1.000 & 0.953 & 0.405 & 0.022 & 0 & 0 & 0 \\
\hline ADF: Rejected $H_{0}^{\prime}$ & 1.000 & 1.000 & 1.000 & 1.000 & 0.976 & 0.561 & 0.188 & 0.073 \\
\hline PP: Rejected $H_{0}^{\prime}$ & 1.000 & 1.000 & 1.000 & 1.000 & 1.000 & 0.803 & 0.184 & 0.049 \\
\hline $\begin{array}{l}\mathrm{N}=5000 \\
X^{(d, 0,1)}\end{array}$ & $=-0.2$ & $\boldsymbol{d}=\mathbf{0}$ & $d=0.2$ & $d=0.4$ & $d=0.6$ & $d=0.8$ & $d=1$ & $d=1.2$ \\
\hline$\tilde{S}_{N}:$ Reject & 0 & 0 & 0 & 0 & 0.933 & 1.000 & 1.000 & 1.000 \\
\hline KPSS: Rejected $H_{0}$ & 0 & 0.082 & 0.689 & 0.970 & 1.000 & 1.000 & 1.000 & 1.000 \\
\hline$V / S:$ Rejected $H_{0}$ & 0 & 0.075 & 0.723 & 0.970 & 0.996 & 1.000 & 1.000 & 1.000 \\
\hline$\tilde{T}_{N}^{\prime}:$ Rejected $H_{0}^{\prime}$ & 1.000 & 1.000 & 1.000 & 0.940 & 0 & 0 & 0 & 0 \\
\hline ADF: Rejected $H_{0}^{\prime}$ & 1.000 & 1.000 & 1.000 & 1.000 & 1.000 & 0.753 & 0.124 & 0.086 \\
\hline PP: Rejected $H_{0}^{\prime}$ & 1.000 & 1.000 & 1.000 & 1.000 & 1.000 & 0.918 & 0.109 & 0.139 \\
\hline
\end{tabular}


We applied the fractional unit roots tests $\tilde{F}_{N}, \widehat{T}_{L V 1}$ and $\widehat{T}_{L V 10}$ to several fractional processes and displayed the results in Table 9 . As it is a test specially devoted to FARIMA $(0, d, 0)$ processes, we only applied the fractional unit roots test $\widehat{T}_{D G M}$ to those processes. Finally, note that we also consider the additional sample size $N=200$ to $N=500$ and $N=5000$ used in other simulations because this could help to better evaluate the power of the several tests (since for $N=500$ and $N=5000$ the test powers are often 1).

Table 9: Comparisons of Fractional Unit Roots tests, as 1000 independent Monte Carlo experiment replications, of processes for several values of $d$ and $N$. Note that the AR parameter of the $\operatorname{ARFIMA}(1, d, 0)$ is 0.5 and the AR and MA parameters of $\operatorname{ARFIMA}(1, d, 1)$ are respectively -0.3 and 0.7 . The accuracy of tests is measured by the rejection probabilities.

\begin{tabular}{lllllll}
\hline $\begin{array}{l}\mathbf{N}=\mathbf{2 0 0} \\
\text { ARFIMA(0, } \boldsymbol{d}, \mathbf{0})\end{array}$ & $\boldsymbol{d}=\mathbf{0 . 5}$ & $\boldsymbol{d}=\mathbf{0 . 6}$ & $\boldsymbol{d}=\mathbf{0 . 7}$ & $\boldsymbol{d}=\mathbf{0 . 8}$ & $\boldsymbol{d}=\mathbf{0 . 9}$ & $\boldsymbol{d}=\mathbf{1}$ \\
\hline$\widetilde{F}_{N}:$ Rejected $H_{0}^{F} U R$ & 0.995 & 0.960 & 0.819 & 0.577 & 0.292 & 0.132 \\
$\widehat{T}_{D G M}:$ Rejected $H_{0}^{F U R}$ & 0.991 & 0.982 & 0.956 & 0.734 & 0.277 & 0.058 \\
$\widehat{T}_{L V 1}:$ Rejected $H_{0}^{F} U R$ & 0.999 & 0.998 & 0.932 & 0.604 & 0.185 & 0.059 \\
$\widehat{T}_{L V 10}:$ Rejected $H_{0}^{F U R}$ & 0.414 & 0.276 & 0.206 & 0.128 & 0.069 & 0.044
\end{tabular}

\section{$\operatorname{ARFIMA}(1, d, 0)$}

$\widetilde{F}_{N}$ : Rejected $H_{0}^{F U R}$

$\widehat{T}_{L V 1}$ : Rejected $H_{0}^{F U R}$

0.998

0.975

0.881

0.653

0.388

0.136

$\widehat{T}_{L V 10}$ : Rejected $H_{0}^{F U R}$

0.433

1.000

1.000

0.992

0.851

0.056

\section{$\operatorname{ARFIMA}(1, d, 1)$}

\begin{tabular}{|c|c|c|c|c|c|c|}
\hline$\widetilde{F}_{N}:$ Rejected $H_{0}^{F} \cup R$ & 0.961 & 0.870 & 0.654 & 0.416 & 0.183 & 0.076 \\
\hline$\widehat{T}_{L V 1}:$ Rejected $H_{0}^{F U R}$ & 0.996 & 0.942 & 0.520 & 0.087 & 0.099 & 0.571 \\
\hline$\widehat{T}_{L V 10}:$ Rejected $H_{0}^{F U R}$ & 0.353 & 0.277 & 0.144 & 0.095 & 0.067 & 0.044 \\
\hline \multicolumn{7}{|l|}{$X^{(d, 0,1)}$} \\
\hline$\widetilde{F}_{N}:$ Rejected $H_{0}^{F U R}$ & 0.993 & 0.956 & 0.825 & 0.569 & 0.318 & 0.125 \\
\hline$\widehat{T}_{L V 1}:$ Rejected $H_{0}^{F U R}$ & 1.000 & 0.999 & 0.965 & 0.716 & 0.358 & 0.106 \\
\hline$\widehat{T}_{L V 10}:$ Rejected $H_{0}^{F U R}$ & 0.682 & 0.504 & 0.294 & 0.173 & 0.110 & 0.057 \\
\hline$N=500$ & $d=0.5$ & $d=0.6$ & $d=0.7$ & $d=0.8$ & $d=0.9$ & $d=1$ \\
\hline \multicolumn{7}{|l|}{$\operatorname{ARFIMA}(0, d, 0)$} \\
\hline$\widetilde{F}_{N}:$ Rejected $H_{0}^{F U R}$ & 0.998 & 0.991 & 0.968 & 0.816 & 0.416 & 0.101 \\
\hline$\widehat{T}_{D G M}:$ Rejected $H_{0}^{F U R}$ & 0.998 & 0.998 & 0.999 & 0.984 & 0.607 & 0.049 \\
\hline$\widehat{T}_{L V 1}:$ Rejected $H_{0}^{F U R}$ & 1.000 & 1.000 & 1.000 & 0.960 & 0.441 & 0.052 \\
\hline$\widehat{T}_{L V 10}:$ Rejected $H_{0}^{F U R}$ & 0.912 & 0.783 & 0.527 & 0.243 & 0.095 & 0.048 \\
\hline
\end{tabular}


Table 9: (continued)

\begin{tabular}{|c|c|c|c|c|c|c|}
\hline \multicolumn{7}{|l|}{$\operatorname{ARFIMA}(1, d, 0)$} \\
\hline$\widetilde{F}_{N}:$ Rejected $H_{0}^{F U R}$ & 0.998 & 0.994 & 0.952 & 0.814 & 0.510 & 0.145 \\
\hline$\widehat{T}_{L V 1}:$ Rejected $H_{0}^{F U R}$ & 1.000 & 1.000 & 1.000 & 1.000 & 0.669 & 0.053 \\
\hline$\widehat{T}_{L V 10}:$ Rejected $H_{0}^{F U R}$ & 0.900 & 0.787 & 0.509 & 0.270 & 0.115 & 0.048 \\
\hline \multicolumn{7}{|l|}{$\operatorname{ARFIMA}(1, d, 1)$} \\
\hline$\widetilde{F}_{N}:$ Rejected $H_{0}^{F U R}$ & 0.999 & 0.988 & 0.904 & 0.619 & 0.241 & 0.088 \\
\hline$\widehat{T}_{L V 1}:$ Rejected $H_{0}^{F U R}$ & 1.000 & 0.998 & 0.927 & 0.150 & 0.135 & 0.919 \\
\hline$\widehat{T}_{L V 10}:$ Rejected $H_{0}^{F U R}$ & 0.891 & 0.699 & 0.416 & 0.220 & 0.101 & 0.040 \\
\hline \multicolumn{7}{|l|}{$X^{(d, 0,1)}$} \\
\hline$\widetilde{F}_{N}:$ Rejected $H_{0}^{F U R}$ & 0.999 & 0.990 & 0.937 & 0.800 & 0.421 & 0.109 \\
\hline$\widehat{T}_{L V 1}:$ Rejected $H_{0}^{F U R}$ & 1.000 & 1.000 & 1.000 & 0.987 & 0.638 & 0.132 \\
\hline$\widehat{T}_{L V 10}:$ Rejected $H_{0}^{F U R}$ & 0.981 & 0.902 & 0.635 & 0.358 & 0.170 & 0.072 \\
\hline $\mathbf{N}=\mathbf{5 0 0 0}$ & $d=0.5$ & $d=0.6$ & $d=0.7$ & $d=0.8$ & $d=0.9$ & $d=1$ \\
\hline \multicolumn{7}{|l|}{$\operatorname{ARFIMA}(0, d, 0)$} \\
\hline$\widetilde{F}_{N}:$ Rejected $H_{0}^{F U R}$ & 1.000 & 1.000 & 1.000 & 0.996 & 0.941 & 0.145 \\
\hline$\widehat{T}_{D G M}:$ Rejected $H_{0}^{F U R}$ & 1.000 & 1.000 & 1.000 & 1.000 & 1.000 & 0.059 \\
\hline$\widehat{T}_{L V 1}:$ Rejected $H_{0}^{F U R}$ & 1.000 & 1.000 & 1.000 & 1.000 & 1.000 & 0.055 \\
\hline$\widehat{T}_{L V 10}:$ Rejected $H_{0}^{F U R}$ & 1.000 & 1.000 & 1.000 & 0.997 & 0.576 & 0.049 \\
\hline \multicolumn{7}{|l|}{$\operatorname{ARFIMA}(1, d, 0)$} \\
\hline$\widetilde{F}_{N}:$ Rejected $H_{0}^{F U R}$ & 1.000 & 1.000 & 0.998 & 0.994 & 0.904 & 0.105 \\
\hline$\widehat{T}_{L V 1}:$ Rejected $H_{0}^{F U R}$ & 1.000 & 1.000 & 1.000 & 1.000 & 1.000 & 0.054 \\
\hline$\widehat{T}_{L V 10}:$ Rejected $H_{0}^{F U R}$ & 1.000 & 1.000 & 1.000 & 0.999 & 0.652 & 0.056 \\
\hline \multicolumn{7}{|l|}{$\operatorname{ARFIMA}(1, d, 1)$} \\
\hline$\widetilde{F}_{N}:$ Rejected $H_{0}^{F U R}$ & 1.000 & 1.000 & 0.998 & 0.989 & 0.808 & 0.072 \\
\hline$\widehat{T}_{L V 1}:$ Rejected $H_{0}^{F U R}$ & 1.000 & 1.000 & 1.000 & 0.884 & 0.965 & 1.000 \\
\hline$\widehat{T}_{L V 10}:$ Rejected $H_{0}^{F U R}$ & 1.000 & 1.000 & 1.000 & 0.988 & 0.488 & 0.051 \\
\hline \multicolumn{7}{|l|}{$x^{(d, 0,1)}$} \\
\hline$\widetilde{F}_{N}:$ Rejected $H_{0}^{F U R}$ & 1.000 & 1.000 & 1.000 & 0.998 & 0.941 & 0.122 \\
\hline$\widehat{T}_{L V 1}:$ Rejected $H_{0}^{F U R}$ & 1.000 & 1.000 & 1.000 & 1.000 & 0.999 & 0.269 \\
\hline$\widehat{T}_{L V 10}:$ Rejected $H_{0}^{F U R}$ & 1.000 & 1.000 & 1.000 & 0.983 & 0.493 & 0.058 \\
\hline
\end{tabular}

Conclusions of simulations: If $\widehat{T}_{D G M}$ and $\widehat{T}_{L V 1}$ provide extremely convincing results for $\operatorname{ARFIMA}(0, d, 0)$ processes, $\widehat{T}_{L V 1}$ is still very accurate for ARFIMA $(1, d, 0)$ processes. From its definition, the fractional unit $\widehat{T}_{L V 1}$ roots can not be used fruitfully for $\operatorname{ARFIMA}(1, d, 1)$ or $X^{(d, 0,1)}$ processes but it can clearly be applied to a more general class of processes than $\widehat{T}_{D G M}$. 
The same for $\widehat{T}_{L V 10}$ which can be applied likely to a more general class of processes than $\widehat{T}_{L V 1}$. However, if the results obtained here for $\operatorname{ARFIMA}(1, d, 1)$ or $X^{(d, 0,1)}$ are satisfying and indicate that this test could be applied when $N=500$ and $N=5000$, this is not the case for $N=200$ for all the considered processes because this test requires the estimation of too many parameters. Moreover, $\widehat{T}_{L V 10}$ can not theoretically be applied to $\operatorname{ARFIMA}(1, d, 1)$ or $\mathrm{X}^{(d, 0,1)}$ processes and additional simulations realized with $N=500000$ indicate a rejection probability $\simeq 0.16$ for $\operatorname{ARFIMA}(1, d, 1)$ processes when $d=1$ (instead of 0.05$)$. However, when $N=500000$, a user could probably chose $\widehat{T}_{L V 20}$ or $\widehat{T}_{L V 50}$ which would provide satisfying results.

The fractional unit roots test $\widetilde{F}_{N}$ constructed from $\widetilde{d}_{N}^{(M I R)}$ does not have these drawbacks: this is a data-driven test and it can be applied to a large family of fractional processes. Hence, the results of simulations obtained with $\widetilde{F}_{N}$ are satisfying (even if they are less efficient for specific processes than those obtained with $\widehat{T}_{L V 10}$ which requires the knowledge of the $A R$ component). Even if this is a semiparametric test, the results obtained for $N=200$ are reasonable. However, note that the rejection probability of $\tilde{F}_{N}$ for $d=0.9$ is much bigger than for $d=1$ when $N=200$ and $N=500$. Hence one could size adjust this test to get a better performance.

\section{Conclusion}

The adaptive data-driven memory parameter estimator $\widetilde{d}_{N}^{(M I R)}$ proposed in this paper has a lot of advantages. Firstly, for any process belonging to the set $(\operatorname{IG}(d, \beta))_{-0.5<d<1.25,0<\beta \leq 2}$, it follows a CLT with a convergence rate reaching the minimax convergence rate (up to a multiplicative logarithm term) and this CLT is obtained without any choice of bandwidth or trimming parameter. Secondly, the numerical performances of this estimator are often better than those of the most accurate semiparametric memory parameter estimators, especially in case of trended processes (the robustness of IR estimator was already established in Surgailis et al. 2008). Finally, data-driven stationarity and fractional unit roots tests are constructed from this estimator and they provide accurate competitive results with respect to classical unit roots or fractional unit roots tests. Improving the performance of those tests could be an interesting task.

An asymptotic study of these new estimator and tests for linear processes could be an interesting extension of this paper. However, this requires to proof a multidimensional CLT theorem for a non-polynomial function of a multidimensional linear process which is a difficult result to be established. 


\section{Proofs}

Two technical lemmas are first established:

Lemma 7.1: For all $\lambda>0$

1. For $a \in(0,2), \frac{2}{|\lambda|^{a-1}} \int_{0}^{\infty} \frac{\sin (\lambda x)}{x^{a}} d x=\frac{4 a}{2^{a}|\lambda|^{a}} \int_{0}^{\infty} \frac{\sin ^{2}(\lambda x)}{x^{a+1}} d x=\frac{\pi}{\Gamma(a) \sin \left(\frac{a \pi}{2}\right)}$;

2. For $b \in(-1,1), \frac{1}{2^{1-b}-1} \int_{0}^{\infty} \frac{\sin ^{4}(\lambda x)}{x^{4-b}} d x=\frac{16}{-15+6 \cdot 2^{3-b}-3^{3-b}} \times \int_{0}^{\infty} \frac{\sin ^{6}(\lambda x)}{x^{4-b}} d x=\frac{2^{3-b}|\lambda|^{3-b} \pi}{4 \Gamma(4-b) \sin \left(\frac{(1-b) \pi}{2}\right)}$;

3. For $b \in(1,3), \frac{1}{1-2^{1-b}} \int_{0}^{\infty} \frac{\sin ^{4}(\lambda x)}{x^{4-b}} d x=\frac{16}{15+6 \cdot 2^{3-b}+3^{3-b}} \times \int_{0}^{\infty} \frac{\sin ^{6}(\lambda x)}{x^{4-b}} d x=\frac{2^{3-b}|\lambda|^{3-b} \pi}{4 \Gamma(4-b) \sin \left(\frac{(3-b) \pi}{2}\right)}$.

Proof. These equations are given or deduced (using decompositions of $\sin ^{j}$ (.) and integration by parts) from (see Doukhan et al., p. 31).

Lemma 7.2: For $j-4,6$, denote

$$
J_{j}(a, m):=\int_{0}^{\pi} x^{a} \frac{\sin ^{j}\left(\frac{m x}{2}\right)}{\sin ^{4}\left(\frac{x}{2}\right)} d x .
$$

Then, we have the following expansions when $m \rightarrow \infty$ :

$$
J_{j}(a, m)= \begin{cases}C_{j 1}(a) m^{3-a}+O\left(m^{1-a}\right) & \text { if }-1<a<1 \\ C_{j 1}^{\prime}(1) m^{3-a}+O(\log (m)) & \text { if } a=1 \\ C_{j 1}^{\prime}(a) m^{3-a}+O(1) & \text { if } 1<a<3 \\ C_{j 2}^{\prime}(3) \log (m)+O(1) & \text { if } a=3 \\ C^{\prime \prime}{ }_{j 1}(a)+O\left(m^{-((a-3) \hat{2})}\right) & \text { if } a>3\end{cases}
$$

with the following real constants (which do not vanish for any a on the corresponding set):

$$
\begin{aligned}
& -\quad C_{41}(a):=\frac{4 \pi\left(1-\frac{2^{3-a}}{4}\right)}{(3-a) \Gamma(3-a) \sin \left(\frac{(3-a) \pi}{2}\right)} \text { and } C_{61}(a):=\frac{\pi\left(15-6 \cdot 2^{3-a}+3^{3-a}\right)}{4(3-a) \Gamma(3-a) \sin \left(\frac{(3-a) \pi}{2}\right)} \\
& -\quad C_{41}^{\prime}(a):=\left(\frac{6}{3-a} 1_{\{1 \leq a<3\}}+16 \int_{0}^{1} \frac{\sin ^{4}\left(\frac{y}{2}\right)}{y^{4-a}} d y+2 \int_{1}^{\infty} \frac{1}{y^{4-a}}(-4 \cos (y)+\cos (2 y)) d y\right) \\
& \quad \text { and } C_{61}^{\prime}(a):=\left[16 \int_{0}^{1} \frac{\sin ^{6}\left(\frac{y}{2}\right)}{y^{4-a}} d y+\frac{5}{3-a} 1_{\{1 \leq a<3\}}+\right. \\
& \left.\quad \frac{1}{2} \int_{1}^{\infty} \frac{1}{y^{4-a}}(-15 \cos (y)+6 \cos (2 y)-\cos (3 y)) d y\right] \\
& -\quad C_{42}^{\prime}(a):=\left(6 \cdot 1_{\{a=3\}}+1_{\{a=1\}}\right) \text { and } C_{62}^{\prime}(a):=\left(5 \cdot 1_{\{a=3\}}+\frac{5}{6} \cdot 1_{\{a=1\}}\right) \\
& -\quad C^{\prime \prime}{ }_{41}(a):=\frac{3}{8} \int_{0}^{\pi} \frac{x^{a}}{\sin ^{4}\left(\frac{x}{2}\right)} d x \text { and } C^{\prime \prime}{ }_{61}(a):=\frac{5}{16} \int_{0}^{\pi} \frac{x^{a}}{\sin ^{4}\left(\frac{x}{2}\right)} d x .
\end{aligned}
$$


Proof. The proof of these expansions follows the steps than those of Lemma 5.1 in Bardet and Dola (2012). Hence we write for $j=4,6$,

$$
J_{j}(a, m)=\tilde{J}_{j}(a, m)+\int_{0}^{\pi} x^{a} \sin ^{j}\left(\frac{m x}{2}\right) \frac{1}{\left(\frac{x}{2}\right)^{4}} d x+\int_{0}^{\pi} x^{a} \sin ^{j}\left(\frac{m x}{2}\right) \frac{2}{3} \frac{1}{\left(\frac{x}{2}\right)^{2}} d x
$$

With

$$
\widetilde{J}_{j}(a, m):=\int_{0}^{\pi} x^{a} \sin ^{j}\left(\frac{m x}{2}\right)\left(\frac{1}{\sin ^{4}\left(\frac{x}{2}\right)}-\frac{1}{\left(\frac{x}{2}\right)^{4}}-\frac{2}{3} \frac{1}{\left(\frac{x}{2}\right)^{2}}\right) d x
$$

The expansions when $m \rightarrow \infty$ of both the right hand sided integrals in eq. [7.3] are obtained from Lemma 7.1. It remains to obtain the expansion of $\widetilde{J}_{j}(a, m)$. Then, using classical trigonometric and Taylor expansions:

$$
\begin{aligned}
\sin ^{4}\left(\frac{y}{2}\right)= & \frac{1}{8}(3-4 \cos (y)+\cos (2 y)) \text { and } \frac{1}{\sin ^{4}(y)}-\frac{1}{y^{4}}-\frac{2}{3} \frac{1}{y^{2}} \sim \frac{11}{45}(y \rightarrow 0) \\
\sin ^{6}\left(\frac{y}{2}\right)= & \frac{1}{32}(10-15 \cos (y)+6 \cos (2 y)-\cos (3 y)) \\
& \text { and } \frac{1}{y^{5}}+\frac{1}{3} \frac{1}{y^{3}}-\frac{\cos (y)}{\sin ^{5}(y)} \sim \frac{31}{945} y \quad(y \rightarrow 0),
\end{aligned}
$$

the expansions of $\tilde{J}_{j}(a, m)$ can be obtained.

Numerical experiments show that $C_{41}^{\prime \prime}(a) \neq 0, C_{61}^{\prime \prime}(a) \neq 0, C_{42}^{\prime \prime}(a) \neq 0$ and $C_{62}^{\prime \prime}(a) \neq 0$.

Proof of Proposition 1: This proposition is based on results of Surgailis et al. (2008) and was already proved in Bardet et Dola (2012) in the case $-0.5<d<0.5$.

Mutatis mutandis, the case $0.5<d<1.25$ can be treated exactly following the same steps.

The only new proof which has to be established concerns the case $d=0.5$ since Surgailis et al. (2008) do not provide a CLT satisfied by the (unidimensional) statistic $I R_{N}(m)$ in this case. Let $Y_{m}(j)$ the standardized process defined Surgailis et al. (2008). Then, for $d=0.5$,

$$
\forall j \geq 1, \quad\left|\gamma_{m}(j)\right|=\left|\mathrm{E}\left(Y_{m}(j) Y_{m}(0)\right)\right|=\frac{2}{V_{m}^{2}}\left|\int_{0}^{\pi} \cos (j x) x\left(c_{0}+O\left(x^{\beta}\right)\right) \frac{\sin ^{4}\left(\frac{m x}{2}\right)}{\sin ^{4}\left(\frac{x}{2}\right)} d x\right| .
$$

Denote $\gamma_{m}(j)=\rho_{m}(j)=\frac{2}{V_{m}^{2}}\left(I_{1}+I_{2}\right)$ as in eq. [5.39] of Surgailis et al. (2008). The expansion [2.20] of Surgailis et al. (2008) remains true for $d=0.5$ and therefore $V_{m}^{2} \sim c_{0} V(0.5) m^{2}$ when $m \rightarrow \infty$. The same for the inequality eq. [5.42] when $d=0.5$ 
and thus $\left|I_{1}\right| \leq C m^{4} j^{-2}$. Finally, when $d=0.5$, we still have $I_{2}=j^{-1} \sum_{q=1}^{j / 2} I_{2}(q)$ with $\left|I_{2}(q)\right| \leq C m^{4} j^{-1}$ when $1 \leq q \leq j / m$ and $\left|I_{2}(q)\right| \leq C q^{-4} j^{3}$ when $j / m \leq q \leq j$ (see details p. 536-537 of Surgailis et al. 2008). Then, the inequality eq. [5.41] remains true for $d=0.5$ and since we consider here $j \geq m$,

$$
\left|I_{2}\right| \leq C m^{3} j^{-1} \Rightarrow\left|I_{1}+I_{2}\right| \leq C m^{3} j^{-1} \Rightarrow\left|\gamma_{m}(j)\right|=\left|\rho_{m}(j)\right| \leq \frac{2}{V_{m}^{2}}\left(\left|I_{1}+I_{2}\right|\right) \leq C \frac{m}{j} .
$$

Now let $\eta_{m}(j):=\frac{\left|Y_{m}(j)+Y_{m}(j+m)\right|}{\left|Y_{m}(j)\right|+\left|Y_{m}(j+m)\right|}:=\psi\left(Y_{m}(j), Y_{m}(j+m)\right)$. The Hermite rank of the function $\psi$ is 2 and therefore the eq. [5.23] of Surgailis et al. (2008) obtained from Lemma 1 of Arcones (1994) remains valid. Hence:

$$
\mid \operatorname{Cov}\left(\eta_{m}(0),\left(\eta_{m}(j)\right) \mid \leq C \frac{m^{2}}{j^{2}},\right.
$$

and then the eqs [5.28]-[5.31] of Surgailis et al. (2008) remain valid for all $d \in[0.5,1.25)$. Then for $d=0.5$,

$$
\sqrt{\frac{N}{m}}\left(I R_{N}(m)-\mathrm{E}\left[I R_{N}(m)\right]\right) \underset{[N / m] \wedge m \rightarrow \infty}{\stackrel{\mathcal{L}}{\rightarrow}} \mathcal{N}\left(0, \sigma^{2}(0.5)\right),
$$

with $\sigma^{2}(0.5) \simeq(0.2524)^{2}$.

To establish the multidimensional CLT for $0.5 \leq d \leq 1.25$ from eq. [7.4] and unidimensional CLT of Surgailis et al. (2008) for $0.5<d \leq 1.25$, we can reproduce exactly the steps 1 and 2 of the proof in Proposition 2.1 of Bardet and Dola (2012).

Proof of Property 1: As in Surgailis et al. (2008), we can write:

$$
\mathrm{E}\left[\operatorname{IR}_{N}(m)\right]=\mathrm{E} \frac{\left|Y^{0}+Y^{1}\right|}{\left|Y^{0}\right|+\left|Y^{1}\right|}=\wedge\left(\frac{R_{m}}{V_{m}^{2}}\right) \text { with } \frac{R_{m}}{V_{m}^{2}}:=1-2 \frac{\int_{0}^{\pi} f(x) \frac{\sin ^{6}\left(\frac{m x}{2}\right)}{\sin ^{2}\left(\frac{x}{2}\right)} d x}{\int_{0}^{\pi} f(x) \frac{\sin ^{4}\left(\frac{m x}{2}\right)}{\sin ^{2}\left(\frac{x}{2}\right)} d x}
$$

Therefore an expansion of $R_{m} / V_{m}^{2}$ provides an expansion of $\mathrm{E}\left[\operatorname{IR}_{N}(m)\right]$ when $m \rightarrow \infty$.

Step 1: Let $f$ satisfy Assumption $I G(d, \beta)$. Then we are going to establish that there exist positive real numbers $C_{1}, C_{2}$, and $C_{3}$ specified in eqs [7.5], [7.6] and [7.7] such that for $0.5 \leq d<1.5$ and with $\rho(d)$ defined in eq. [2.6],

1. If $\beta<2 d-1, \frac{R_{m}}{V_{m}^{2}}=\rho(d)+C_{1}(2-2 d, \beta) m^{-\beta}+O\left(m^{-2}+m^{-2 \beta}\right)$;

2. If $\beta=2 d-1, \frac{R_{m}}{V_{m}^{2}}=\rho(d)+C_{2}(2-2 d, \beta) m^{-\beta}+O\left(m^{-2}+m^{-2-\beta} \log (m)+m^{-2 \beta}\right)$; 
3. If $2 d-1<\beta<2 d-1, \frac{R_{m}}{V_{m}^{2}}=\rho(d)+C_{3}(2-2 d, \beta) m^{-\beta}+O\left(m^{-\beta-\varepsilon}+m^{-2 d-1} \log (m)\right.$ $\left.+m^{-2 \beta}\right)$

4. If $\beta=2 d-1, \frac{R_{m}}{V_{m}^{2}}=\rho(d)+O\left(m^{-2 d-1} \log (m)+m^{-2}\right)$.

Under Assumption $I G(d, \beta)$ and with $J_{\mathrm{j}}(a, m)$ defined in eq. [7.2] in Lemma 7.2, it is clear that,

$$
\frac{R_{m}}{V_{m}^{2}}=1-2 \frac{J_{6}(2-2 d, m)+\frac{c_{1}}{c_{0}} J_{6}(2-2 d+\beta, m)+O\left(J_{6}(2-2 d+\beta+\varepsilon)\right)}{J_{4}(2-2 d, m)+\frac{c_{1}}{c_{0}} J_{4}(2-2 d+\beta, m)+O\left(J_{4}(2-2 d+\beta+\varepsilon)\right)},
$$

since $\int_{0}^{\pi} O\left(x^{2-2 d+\beta+\varepsilon}\right) \frac{\sin ^{j}\left(\frac{m x}{2}\right)}{\sin ^{2}\left(\frac{x}{2}\right)} d x=O\left(J_{j}(2-2 d+\beta+\varepsilon)\right)$. Now using the results of Lemma 7.2 and constants $C_{j \ell}, C_{j \ell}^{\prime}$ and $C_{j l}^{\prime \prime} j=4,6, \ell=1,2$ defined in Lemma 7.2, 1. Let $0<\beta<2 d-1<2$, i. e. $-1<2-2 d+\beta<1$. Then

$$
\begin{aligned}
\frac{R_{m}}{V_{m}^{2}}= & 1-2 \frac{C_{61}(2-2 d) m^{1+2 d}+O\left(m^{2 d-1}\right)+\frac{c_{1}}{c_{0}} C_{61}(2-2 d+\beta) m^{1+2 d-\beta}+O\left(m^{2 d-1-\beta}\right)}{C_{41}(2-2 d) m^{1+2 d}+O\left(m^{2 d-1}\right)+\frac{c_{1}}{c_{0}} C_{41}(2-2 d+\beta) m^{1+2 d-\beta}+O\left(m^{2 d-1-\beta}\right)} \\
= & 1-\frac{2}{C_{41}(2-2 d)}\left[C_{16}(2-2 d)+\frac{c_{1}}{C_{0}} C_{61}(2-2 d+\beta) m^{-\beta}\right] \\
& {\left[1-\frac{c_{1}}{c_{0}} \frac{C_{41}(2-2 d+\beta)}{C_{41}(2-2 d)} m^{-\beta}\right]+O\left(m^{-2}\right) } \\
= & 1-\frac{2 C_{61}(2-2 d)}{C_{41}(2-2 d)}+2 \frac{c_{1}}{C_{0}}\left[\frac{C_{61}(2-2 d) C_{41}(2-2 d+\beta)}{C_{41}(2-2 d) C_{41}(2-2 d)}-\frac{C_{61}(2-2 d+\beta)}{C_{41}(2-2 d)}\right] m^{-\beta} \\
& +O\left(m^{-2}+m^{-2 \beta}\right) .
\end{aligned}
$$

As a consequence,

$$
\begin{aligned}
& \frac{R_{m}}{V_{m}^{2}}=\rho(d)+C_{1}(2-2 d, \beta) m^{-\beta}+O\left(m^{-2}+m^{-2 \beta}\right)(m \rightarrow \infty) \text {, with } 0<\beta<2 d-1<2 \text { and } \\
& C_{1}(2-2 d, \beta):=2 \frac{c_{1}}{C_{0}} \frac{1}{C_{41}^{2}(2-2 d)}\left[C_{61}(2-2 d) C_{41}(2-2 d+\beta)-C_{61}(2-2 d+\beta) C_{41}(2-2 d)\right],
\end{aligned}
$$

and numerical experiments proves that $C_{1}(2-2 d, \beta) / c_{1}$ is negative for any $d \in(0.5,1.5)$ and $\beta>0$. 
2. Let $\beta=2 d-1$, i.e. $2-2 d+\beta=1$. Then,

$$
\begin{aligned}
\frac{R_{m}}{V_{m}^{2}}=1 & -2 \frac{C_{61}(2-2 d) m^{1+2 d}+O\left(m^{2 d-1}\right)+\frac{c_{1}}{C_{0}} C_{61}^{\prime}(1) m^{1-2 d}+O(\log (m))}{C_{41}(2-2 d) m^{1+2 d}+O\left(m^{2 d-1}\right)+\frac{C_{1}}{c_{0}} C_{41}^{\prime}(1) m^{1-2 d}+O(\log (m))} \\
= & 1-\frac{2}{C_{41}(2-2 d)}\left[C_{61}(2-2 d)+\frac{C_{1}}{C_{0}} C_{61}^{\prime}(1) m^{1-2 d}\right]\left[1-\frac{c_{1}}{C_{0}} \frac{C^{\prime}{ }_{41}^{\prime}(1)}{C_{41}(2-2 d)} m^{1-2 d}\right] \\
& +O\left(m^{-2}+m^{-2 d-1} \log (m)\right) \\
=1 & -\frac{2 C_{61}(2-2 d)}{C_{41}(2-2 d)}+2 \frac{C_{1}}{C_{0}}\left[\frac{C_{61}(2-2 d) C_{41}^{\prime}(1)}{C_{41}(2-2 d) C_{41}(2-2 d)}-\frac{C_{61}^{\prime}(1)}{C_{41}(2-2 d)}\right] m^{1-2 d} \\
& +O\left(m^{-2}+m^{-2 d-1} \log (m)+m^{2-4 d}\right) .
\end{aligned}
$$

As a consequence,

$$
\begin{aligned}
& \frac{R_{m}}{V_{m}^{2}}=\rho(d)+C_{2}(2-2 d, \beta) m^{-\beta}+O\left(m^{-2}+m^{-2-\beta} \log (m)+m^{-2 \beta}\right) \quad(m \rightarrow \infty) \\
& \quad \text { with } 0<\beta=2 d-1<2 \text { and } \\
& C_{2}(2-2 d, \beta):=2 \frac{C_{1}}{C_{0}} \frac{1}{C_{41}^{2}(2-2 d)}\left[C_{61}(2-2 d) C_{41}^{\prime}(1)-C_{61}^{\prime}(1) C_{41}(2-2 d)\right],
\end{aligned}
$$

and numerical experiments proves that $C_{2}(2-2 d, \beta) / c_{1}$ is negative for any $d \in[0.5,1.5)$ and $\beta>0$.

3. Let $2 d-1<\beta<2 d+1$, i.e. $1<2-2 d+\beta<3$. Then,

$$
\begin{aligned}
\frac{R_{m}}{V_{m}^{2}}= & 1-2 \frac{C_{61}(2-2 d) m^{1+2 d}+\frac{c_{1}}{c_{0}} C_{61}^{\prime}(2-2 d+\beta) m^{1+2 d-\beta}+O\left(m^{1+2 d-\beta-\epsilon}+\log (m)\right)}{C_{41}(2-2 d) m^{1+2 d}+\frac{c_{1}}{c_{0}} C_{41}^{\prime}(2-2 d+\beta) m^{1+2 d-\beta}+O\left(m^{1+2 d-\beta-\epsilon}+m^{-2 d-1} \log (m)\right)} \\
= & 1-\frac{2}{C_{41}(2-2 d)}\left[C_{61}(2-2 d)+\frac{c_{1}}{C_{0}} C_{61}^{\prime}(2-2 d+\beta) m^{-\beta}\right] \\
& {\left[1-\frac{c_{1}}{C_{0}} \frac{C_{41}^{\prime}(2-2 d+\beta)}{C_{41}(2-2 d)} m^{-\beta}\right]+O\left(m^{-\beta-\epsilon}+m^{-2 d-1} \log (m)\right) } \\
= & 1-\frac{2 C_{61}(2-2 d)}{C_{41}(2-2 d)}+2 \frac{c_{1}}{C_{0}}\left[\frac{C_{61}(2-2 d) C_{41}^{\prime}(2-2 d+\beta)}{C_{41}(2-2 d) C_{41}(2-2 d)}-\frac{C_{61}^{\prime}(2-2 d+\beta)}{C_{41}(2-2 d)}\right] m^{-\beta} \\
& +O\left(m^{-\beta-\varepsilon}+m^{-2 d-1} \log (m)\right) .
\end{aligned}
$$

As a consequence, 


$$
\begin{aligned}
& \frac{R_{m}}{V_{m}^{2}}=\rho(d)+C_{3}(2-2 d, \beta) m^{-\beta}+O\left(m^{-\beta-\varepsilon}+m^{-2 d-1} \log (m)+m^{-2 \beta}\right)(m \rightarrow \infty), \text { and } \\
& \quad C_{3}(2-2 d, \beta):=2 \frac{c_{1}}{c_{0}} \frac{1}{C_{41}^{2}(2-2 d)}\left[C_{61}(2-2 d) C_{41}^{\prime}(2-2 d+\beta)-C_{61}^{\prime}(2-2 d+\beta) C_{41}(2-2 d)\right],
\end{aligned}
$$

and numerical experiments proves that $C_{3}(2-2 d, \beta) / c_{1}$ is negative for any $d \in[0.5$, 1.5) and $\beta>0$.

4. Let $\beta=2 d+1$. Then, Once again with Lemma 7.2

$$
\begin{aligned}
\frac{R_{m}}{V_{m}^{2}}= & 1-2 \frac{C_{61}(2-2 d) m^{1+2 d}+O\left(m^{2 d-1}\right)+\frac{c_{1}}{c_{0}} C_{62}^{\prime}(3) \log (m)+O(1)}{C_{41}(2-2 d) m^{1+2 d}+O\left(m^{2 d-1}\right)+\frac{C_{1}}{c_{0}} C_{42}^{\prime}(3) \log (m)+O(1)} \\
= & 1-\frac{2}{C_{41}(2-2 d)}\left[C_{61}(2-2 d)+\frac{C_{1}}{C_{0}} C_{62}^{\prime}(3) m^{-\beta} \log (m)\right] \\
& {\left[1-\frac{c_{1}}{C_{0}} \frac{C_{42}^{\prime}(3)}{C_{41}(2-2 d)} m^{-\beta} \log (m)\right]+O\left(m^{-2}+m^{-2 d-1}\right) } \\
= & 1-\frac{2 C_{61}(2-2 d)}{C_{41}(2-2 d)}+2 \frac{C_{1}}{C_{0}}\left[\frac{C_{61}(2-2 d) C_{42}^{\prime}(3)}{C_{41}(2-2 d) C_{41}(2-2 d)}-\frac{C_{62}^{\prime}(3)}{C_{41}(2-2 d)}\right] m^{-\beta} \log (m)+O\left(m^{-2}\right) .
\end{aligned}
$$

As a consequence,

$$
\frac{R_{m}}{V_{m}^{2}}=\rho(d)+O\left(m^{-2 d-1} \log (m)+m^{-2}\right) \quad(m \rightarrow \infty), \text { with } 2<\beta=2 d+1<4 .
$$

Step 2: A Taylor expansion of $\Lambda(\cdot)$ around $\rho(d)$ provides:

$$
\Lambda\left(\frac{R_{m}}{V_{m}^{2}}\right) \simeq \Lambda(\rho(d))+\left[\frac{\partial \Lambda}{\partial \rho}\right](\rho(d))\left(\frac{R_{m}}{V_{m}^{2}}-\rho(d)\right)+\frac{1}{2}\left[\frac{\partial^{2} \Lambda}{\partial \rho^{2}}\right](\rho(d))\left(\frac{R_{m}}{V_{m}^{2}}-\rho(d)\right)^{2}
$$

Note that numerical experiments show that $\left[\frac{\partial \Lambda}{\partial \rho}\right](\rho)>0.2$ for any $\rho \in(-1,1)$. As a consequence, using the previous expansions of $R_{m} / V_{m}^{2}$ obtained in Step 1 and since $\mathrm{E}\left[I R_{N}(m)\right]=\Lambda\left(R_{m} / V_{m}^{2}\right)$ then for all $0<\beta<2$ :

$$
\begin{aligned}
& \mathrm{E}\left[I R_{N}(m)\right]= \\
& \Lambda_{0}(d)+ \begin{cases}{ }_{1} C^{\prime}{ }_{1}(d, \beta) m^{-\beta}+O\left(m^{-2}+m^{-2 \beta}\right) & \text { if } \beta<2 d-1 \\
c_{1} C_{2}^{\prime}(d, \beta) m^{-\beta}+O\left(m^{-2}+m^{-2-\beta} \log m+m^{-2 \beta}\right) & \text { if } \beta=2 d-1 \\
c_{1} C^{\prime}{ }_{3}(d, \beta) m^{-\beta}+O\left(m^{-\beta-\varepsilon}+m^{-2 d-1} \log m+m^{-2 \beta}\right) & \text { if } 2 d-1<\beta<2 d+1 \\
O\left(m^{-2 d-1} \log m+m^{-2}\right) & \text { if } \beta=1+2 d\end{cases}
\end{aligned}
$$


With $C_{\ell}^{\prime}(d, \beta)=\left[\frac{\partial \Lambda}{\partial \rho}\right](\rho(d)) C_{\ell}(2-2 d, \beta)$ for $\ell=1,2,3$ and $C_{\ell}$ defined in eqs [7.5], [7.6] and [7.7].

Proof of Theorem 1: Using Property 1, if $m \simeq C N^{\alpha}$ with $C>0$ and $(1+2 \beta)^{-1}<\alpha<1$ then $\sqrt{N / m}\left(\mathrm{E}\left[\operatorname{IR}_{N}(m)\right]-\Lambda_{0}(d)\right) \underset{N \rightarrow \infty}{\rightarrow} 0$ and it implies that the multidimensional CLT eq. [2.4] can be

$$
\sqrt{\frac{N}{m}}\left(I_{N}\left(m_{j}\right)-\Lambda_{0}(d)\right)_{1 \leq j \leq p} \underset{N \rightarrow \infty}{\stackrel{\mathcal{L}}{\rightarrow}} \mathcal{N}\left(0, \Gamma_{p}(d)\right) .
$$

It remains to apply the Delta-method with the function $\Lambda_{0}^{-1}$ to CLT eq. [7.9]. This is possible since the function $d \rightarrow \Lambda_{0}(d)$ is an increasing function such that $\left(\Lambda_{0}^{\prime}\right)>0$ and $\left(\Lambda_{0}^{-1}\right)^{\prime}\left(\Lambda_{0}(d)\right)=1 / \Lambda_{0}^{-1}(d)>0$ for all $d \in(-0.5,1.5)$. It achieves the proof of Theorem 1.

Proof of Proposition 2: We use the proof of Proposition 2 in Bardet and Dola (2012). Indeed, this proof is only based on the definitions of $\widehat{d}_{N}\left(j N^{\alpha}\right)$, $\widehat{d}_{N}\left(N^{\alpha}\right), \widehat{Q}_{N}(\alpha)$ and $\widehat{\alpha}_{N}$ which are exactly the same here, and on the CLT satisfied by $\left(\widehat{d}_{N}\left(j N^{\alpha}\right)\right)_{1 \leq j \leq p}$ The only difference is that we suppose here $0<\beta \leq 2$ and $0.5 \leq d<1.25$ instead of $0<\beta$ and $-0.5 \leq d<0.5$ in Bardet and Dola (2012). Then we consider only the case where $\beta<2 d+1$ and $\alpha^{\star}=\frac{1}{(1+2 \beta) \wedge(4 d+3)}=\frac{1}{(1-2 \beta)}$ in our framework.

Proof of Theorem 2: This proof is exactly the same as the proof of Theorem 2 in Bardet and Dola (2012).

Acknowledgments: The authors are grateful to both the referees for their very careful reading and many relevant suggestions and corrections that strongly improve the content and the form of the paper.

\section{References}

Abadir, K. M., W. Distaso, and L. Giraitis. 2007. "Non-Stationarity-Extended Local Whittle Estimation.” Journal of. Econometrics 141:1353-84.

Arcones, M. 1994. "Limit theorems for nonlinear functionals of a stationary Gaussian sequence of vectors.” 1994. Annals of Probability 22:2242-74.

Banerjee, A., J. J. Dolado, J. W. Galbraith, and D. F. Hendry. 1993. Cointegration, Error Correction, and the Econometric Analysis of Non-Stationary Data. Oxford: Oxford University Press. 
Bardet, J. M., and H. Bibi. 2012. "Adaptive Semiparametric Wavelet Estimator and Goodness-ofFit Test for Long-Memory Linear Processes.” Electronic Journal of Statistics 6:2383-419.

Bardet, J. M., and B. Dola. 2012. "Adaptive Estimator of the Memory Parameter and Goodnessof-Fit Test Using a Multidimensional Increment Ratio Statistic." Journal of Multivariate Analysis 105:222-40.

Bardet, J. M., and D. Surgailis. 2011. "Measuring the Roughness of Random Paths by Increment Ratios.” Bernoulli 17:749-80.

Beran, J. 1994. Statistics for Long-Memory Processes. New York: Chapman and Hall.

Breitung, J., and U. Hassler. 2002. "Inference on the Cointegration Rank in Fractionally Integrated Processes.” Journal of Econometrics 110:167-85.

Dolado, J., J. Gonzalo, and L. Mayoral. 2002. “A Fractional Dickey-Fuller Test for Unit Roots.” Econometrica 70 (5):1963-2006.

Dolado, J., J. Gonzalo, and L. Mayoral. 2008. "Wald Tests of I (1) Against I (d) Alternatives: Some New Properties and an Extension to Processes with Trending Components." Studies in Nonlinear Dynamics and Econometrics 12:1-33.

Doukhan, P. Oppenheim, G. and Taqqu M. S., eds. 2003. Theory and Applications of LongRange Dependence. Boston: Birkhäuser.

Elliott, G., T. J. Rothenberg, and J. H. Stock. 1996. "Efficient Tests for an Autoregressive Unit Roots." Econometrica 64:813-36.

Giraitis, L., P. Kokoszka, and R. Leipus. 2001. "Testing for Long-Memory in the Presence of a General Trend." Journal of Applied Probability 38:1033-54.

Giraitis, L., P. Kokoszka, R. Leipus, and G. Teyssiẽ "re. 2003. "Rescaled Variance and Related Tests for Long-Memory in Volatility and Levels." Journal of Econometrics112:265-94.

Giraitis, L., R. Leipus, and A. Philippe. 2006. "A Test for Stationarity Versus Trends and Unit Roots for a Wide Class of Dependent Errors." Econometric Theory 22:989-1029.

Giraitis, L., P. M. Robinson, and A. Samarov. 1997. "Rate Optimal Semi-Parametric Estimation of the Memory Parameter of the Gaussian Time Series with Long Range Dependence.” Journal of Time Series Analysis 18:49-61.

Giraitis, L., P. M. Robinson, and A. Samarov. 2000. "Adaptive Semiparametric Estimation of the Memory Parameter." Journal of Multivariate Analysis 72:183-207.

Giraitis, L., and D. Surgailis. 1990. "A Central Limit Theorem for Quadratic Forms in Strongly Dependent Linear Variables and Its Applications to the Asymptotic Normality of Whittle Estimate." Probability Theory and Related Fields 86:87-104.

Granger, C. W. J., and K. Joyeux. 1980. "An Introduction to Long-Memory Time Series and Fractional Differencing." Journal of Time Series Analysis 1:15-29.

Henry, M. 2007. "Robust Automatic Bandwidth for Long-Memory." In Long Memory in Economics, edited by Teyssière, Gilles, Kirman, Alan, 157-72. Springer-Verlag Berlin Heidelberg: Springer.

Henry, M., and P. M. Robinson. 1996. "Bandwidth Choice in Gaussian Semiparametric Estimation of Long Range Dependence." In Athens Conference on Applied Probability and Time Series Analysis, edited by Robinson, P. M. and Rosenblatt, Murray, Vol. II, 220-232. New York: Springer.

Hurvich, C. M., and W. W. Chen. 2000. "An Efficient Taper for Potentially Over Differenced LongMemory Time Series." Journal of Time Series Analysis 21:155-80.

Hurvich, C. M., and B. K. Ray. 1995. "Estimation of the Memory Parameter for Non-Stationary or Noninvertible Fractionally Integrated Processes.” Journal of Time Series Analysis 16:17-41. 
Iouditsky, A., E. Moulines, and P. Soulier. 2001. "Adaptive Estimation of the Fractional Differencing Coefficient." Bernoulli 7:699-731.

Kew, H., and D. Harris. 2009. "Heteroskedasticity Robust Testing for a Fractional Unit Root." Econometric Theory 25:1734-53.

Kwiatkowski, D., P. C. B. Phillips, P. Schmidt, and Y. Shin. 1992. "Testing the Null Hypothesis of Stationarity Against the Alternative of a Unit Root." Journal of Econometrics 54:159-78. Leybourne, S. J., and B. P. M. McCabe. 1994. "A Consistent Test for a Unit Root," Journal of Business \& Economic Statistics, American Statistical Association, 12:157-166.

Ling, S. 2003. "Adaptive Estimators and Tests of Stationary and Non-Stationary Short and Long Memory ARIMA-GARCH Models." Journal of American Statistical Association 92:1184-92.

Ling, S., and W. K. Li. 2001. "Asymptotic Inference for Non-Stationary Fractionally Integrated Autoregressive Moving-Average Models." Econometric Theory 17:738-65.

Lobato, I., and C. Velasco. 2007. "Efficient Wald Tests for Fractional Unit Roots." Econometrica 75:575-89.

Moulines, E., F. Roueff, and M. S. Taqqu. 2007. "On the Spectral Density of the Wavelet Coefficients of Long Memory Time Series with Application to the Log-Regression Estimation of the Memory Parameter." Journal of Time Series Analysis 28:155-87.

Moulines, E., and P. Soulier. 2003. "Semiparametric Spectral Estimation for Fractional Processes." In Theory and Applications of Long-Range Dependence, edited by P. Doukhan, G. Oppenheim and M. S. Taqqu, 251-301. Boston: Birkhäuser.

Ng, S., and P. Perron. 1996. "Useful Modifications to Some Unit Root Tests with Dependent Errors and Their Local Asymptotic Properties." Review of Economic Studies 63:435-63.

Ng, S., and P. Perron. 2001. "Lag Length Selection and the Construction of the Unit Root Tests with Good Size and Power." Econometrica 69 (6):1519-54.

Nielsen, M. O. 2004. "Efficient Likelihood Inference in Non-Stationary Univariate Models." Econometric Theory 20:116-46.

Phillips, P. C. B. 1999. Discrete Fourier Transforms of Fractional Processes. Technical Report, Yale University.

Phillips, P. C. B., and P. Perron. 1988. "Testing for a Unit Root in Time Series Regression.” Biometrika 75:335-46.

Robinson, P. M.. 1994. "Efficient Tests of Non-Stationary Hypotheses." Journal of the American Statistical Association 89:1420-37.

Robinson, P. M.. 1995a. "Log-Periodogram Regression of Time Series with Long Range Dependence." The Annals of Statistics 23:1048-72.

Robinson, P. M.. 1995b. “Gaussian Semiparametric Estimation of Long Range Dependence." The Annals of Statistics 23:1630-61.

Said, S. E., and D. A. Dickey. 1984. "Testing for Unit Roots in Autoregressive-Moving Average Models of Unknown Order." Biometrika 71:599-607.

Shimotsu, K., and P. C. B. Phillips. 2005. "The Exact Local Whittle Estimation of Fractional Integration." The Annals of Statistics 33:1890-933.

Surgailis, D., G. Teyssière, and M. Vaičiulis. 2008. "The Increment Ratio Statistic." Journal of. Multivariate Analysis 99:510-41.

Tanaka, K. 1999. "The Non-Stationary Fractional Unit Root.” Econometric Theory 15:549-82.

Veitch, D., P. Abry, and M. S. Taqqu. 2003. "On the Automatic Selection of the Onset of Scaling." Fractals 11:377-90. 
Velasco, C. 1999a. "Non-Stationary Log-Periodogram Regression.” Journal of Econometrics 91:325-71.

Velasco, C. 1999b. "Gaussian Semiparametric Estimation of Non-Stationary Time Series." Journal of Time Series Analysis 20:87-127.

Velasco, C., and P. Robinson. 2000. "Whittle Pseudo-Maximum Likelihood Estimation for NonStationary Time Series." Journal of the American Statistical Association 95:1229-43. 